\title{
Anticipated Backward SDEs with Jumps and quadratic-exponential growth drivers *
}

\author{
Masaaki Fujii ${ }^{\dagger} \&$ Akihiko Takahashi ${ }^{\ddagger}$
}

This version: July 9, 2018

\begin{abstract}
In this paper, we study a class of Anticipated Backward Stochastic Differential Equations (ABSDE) with jumps. The solution of the $\operatorname{ABSDE}$ is a triple $(Y, Z, \psi)$ where $Y$ is a semimartingale, and $(Z, \psi)$ are the diffusion and jump coefficients. We allow the driver of the ABSDE to have linear growth on the uniform norm of $Y$ 's future paths, as well as quadratic and exponential growth on the spot values of $(Z, \psi)$, respectively. The existence of the unique solution is proved for Markovian and non-Markovian settings with different structural assumptions on the driver. In the former case, some regularities on $(Z, \psi)$ with respect to the forward process are also obtained.
\end{abstract}

Keywords : predictive mean-field type, time-advanced, quadratic growth, future path dependent driver, ABSDE

\section{Introduction}

As a powerful probabilistic tool to analyze general control problems, non-linear partial differential equations as well as many newly appeared financial problems, backward stochastic differential equations (BSDEs) have attracted strong research interests since the pioneering works of Bismut (1973) [6] and Pardoux \& Peng (1990) [30].

Recently, Peng \& Yang (2009) 32] introduced a new class, so-called anticipated (or timeadvanced) BSDEs, where the drivers are dependent on the conditional expectations of the future paths of the solutions. They originally appeared as adjoint processes when dealing with optimal control problems on delayed systems. Since then various generalizations have been studied by many authors: Oksendal et al. (2011) [28] dealt with a control problem on delayed systems with jumps, Pamen (2015) [29] a stochastic differential game with delay, Xu (2011) [37, Yang \& Elliott (2013) [36] studied some generalizations and conditions for the comparison principle to hold. Jeanblac et al. (2016) [18] studied anticipated BSDEs under a setting of progressive enlargement of filtration. The importance of anticipated BSDEs for financial applications is likely to grow in the coming years because of the set of new regulations (in particular, the margin rule on the independent amount). They require the financial firms to adjust the collateral (or capital) amount based on the expected future maximum loss,

\footnotetext{
${ }^{*}$ Accepted for publication in Stochastics and Dynamics. All the contents expressed in this research are solely those of the author and do not represent any views or opinions of any institutions.

${ }^{\dagger}$ Quantitative Finance Course, Graduate School of Economics, The University of Tokyo. mfujii@e.utokyo.ac.jp

${ }^{\ddagger}$ Quantitative Finance Course, Graduate School of Economics, The University of Tokyo. akihikot@e.utokyo.ac.jp
} 
exposure or the variability of the mark-to-market, which naturally makes the drivers of the pricing BSDEs dependent on the expected future paths of the portfolio values.

In this paper, we are interested in anticipated BSDEs with jumps and quadratic-exponential growth drivers. Although the properties of Lipschitz ABSDEs have been well established, the ABSDEs with quadratic growth generators have not yet appeared in the literature. In addition to the pure mathematical interests, the quadratic growth (exponential growth in the presence of jumps) BSDEs have many applications. In particular, they arise in the context of utility optimization with exponential or power utility functions and the associated indifference valuation, or questions related to risk minimization for the entropic risk measure. They also arise in a class of recursive utilities introduced by Epstein \& Zin (1989) [12] where the investor penalizes the variance of the value function. Their model and its variants have found many applications in economic theory. Once the investor assigns a cost or benefit to the expected value of a future path, which looks almost inevitable due to the new financial regulations, the resultant recursive utility, which corresponds to the driver of the associated BSDE, starts to involve an anticipated component. In this work, we deal with the anticipated BSDEs with jumps of the following form:

$$
Y_{t}=\xi+\int_{t}^{T} \mathbb{E}_{\mathcal{F}_{r}} f\left(r,\left(Y_{v}\right)_{v \in[r, T]}, Y_{r}, Z_{r}, \psi_{r}\right) d r-\int_{t}^{T} Z_{r} d W_{r}-\int_{t}^{T} \int_{E} \psi_{r}(e) \widetilde{\mu}(d r, d e)
$$

where the driver $f(t, \cdot)$ is allowed to have linear growth in $\sup _{v \in[t, T]}\left|Y_{v}\right|$, quadratic in $Z_{t}$ and exponential growth in the jump coefficients $\psi_{t}$. This will be the necessary first step toward the understanding of the general problems involving non-Lipschitz generators with anticipated components and its applications to the various problems mentioned above.

For the (non-anticipated) BSDEs with quadratic growth drivers, the first breakthrough was made by Kobylanski (2000) [23] and then followed by many researchers for its generalization and applications. In the presence of jumps, in particular, they were studied by Becherer (2006) [4, Morlais (2010) [25], Ngoupeyou (2010) [27, Cohen \& Elliott (2015) [7, Kazi-Tani et al. (2015) [21], Antonelli \& Mancini (2016) [1], El Karoui et al. (2016) [10] and Fujii \& Takahashi (2017) [16] with varying generality. An important common tool is the so called

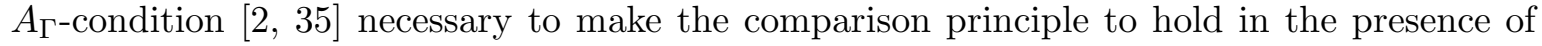
jumps, which is then used to create a monotone sequence of regularized BSDEs.

Although $A_{\Gamma}$-condition is known to hold for the setting of exponential utility optimization [25], it is rather restrictive, and in fact, stronger than the local Lipschitz continuity. Furthermore, in the anticipated settings, the comparison principle does not hold generally

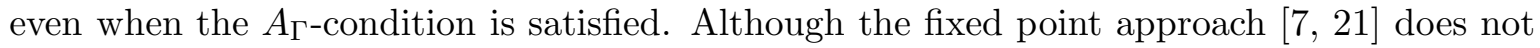
rely on the comparison principle at least for small terminal values, it requires the second-order differentiability of the driver which is difficult to establish in the presence of the general path dependence.

In this paper, we firstly extend the quadratic-exponential structure condition of [3, 10] to allow the dependence on $Y$ 's future paths, and then derive the universal bounds on $(Y, Z, \psi)$ under a general bounded terminal condition. This bounds are then used to prove a stability result under a general non-Markovian setting. Under the Markovian setting, this stability result leads to the compactness result for the deterministic map defined by $u(t, x)=Y_{t}^{t, x}$, which then allows us to prove the existence of the solution in the absence of the $A_{\Gamma^{-}}$-condition. It also provides some regularities on $(Z, \psi)$ with respect to the forward process. As a by product, it makes the $A_{\Gamma^{-}}$-condition unnecessary for the existence, uniqueness and Malliavin's differentiability of quadratic-exponential growth (non-anticipated) BSDEs under the Markovian setting studied in Section 6 of [16. For a non-Markovian setting, we reintroduce the $A_{\Gamma}$-condition and make use of our previous result in [16] to prove the existence of the unique solution. We 
also give a sufficient condition for the comparison principle to hold.

\section{Preliminaries}

\subsection{General Setting}

Let us first state the general setting to be used throughout the paper. $T>0$ is some bounded time horizon. The space $\left(\Omega_{W}, \mathcal{F}_{W}, \mathbb{P}_{W}\right)$ is the usual canonical space for a $d$-dimensional Brownian motion equipped with the Wiener measure $\mathbb{P}_{W}$. We also denote $\left(\Omega_{\mu}, \mathcal{F}_{\mu}, \mathbb{P}_{\mu}\right)$ as a product of canonical spaces $\Omega_{\mu}:=\Omega_{\mu}^{1} \times \cdots \times \Omega_{\mu}^{k}, \mathcal{F}_{\mu}:=\mathcal{F}_{\mu}^{1} \times \cdots \times \mathcal{F}_{\mu}^{k}$ and $\mathbb{P}_{\mu}^{1} \times \cdots \times \mathbb{P}_{\mu}^{k}$ with some constant $k \in \mathbb{N}$, on which each $\mu^{i}$ is a Poisson measure with a compensator $\nu^{i}(d e) d t$. Here, $\nu^{i}(d e)$ is a $\sigma$-finite measure on $\mathbb{R}_{0}=\mathbb{R} \backslash\{0\}$ satisfying $\int_{\mathbb{R}_{0}}|e|^{2} \nu^{i}(d e)<\infty$. For notational simplicity, we write $(E, \mathcal{E}):=\left(\mathbb{R}_{0}^{k}, \mathcal{B}\left(\mathbb{R}_{0}\right)^{k}\right)$. Throughout the paper, we work on the filtered probability space $\left(\Omega, \mathcal{F}, \mathbb{F}=\left(\mathcal{F}_{t}\right)_{t \in[0, T]}, \mathbb{P}\right)$, where the space $(\Omega, \mathcal{F}, \mathbb{P})$ is the product of the canonical spaces $\left(\Omega_{W} \times \Omega_{\mu}, \mathcal{F}_{W} \times \mathcal{F}_{\mu}, \mathbb{P}_{W} \times \mathbb{P}_{\mu}\right)$, and that the filtration $\mathbb{F}=$ $\left(\mathcal{F}_{t}\right)_{t \in[0, T]}$ is the canonical filtration completed for $\mathbb{P}$ and satisfying the usual conditions. In this construction, $\left(W, \mu^{1}, \cdots, \mu^{k}\right)$ are independent. We use a vector notation $\mu(\omega, d t, d e):=$ $\left(\mu^{1}\left(\omega, d t, d e^{1}\right), \cdots, \mu^{k}\left(\omega, d t, d e^{k}\right)\right)$ and denote the compensated Poisson measure as $\widetilde{\mu}:=\mu-\nu$. $\mathbb{F}$-predictable $\sigma$-field on $\Omega \times[0, T]$ is denoted by $\mathcal{P}$. It is well-known that the weak property of predictable representation holds in this setup (see for example [17] chapter XIII).

\subsection{Notation}

We denote a generic constant by $C$ which may change line by line. We write $C=C(a, b, c, \cdots)$ when the constant depends only on the parameters $(a, b, c, \cdots) . \mathcal{T}_{s}^{t}$ denotes the set of $\mathbb{F}$ stopping times $\tau: \Omega \rightarrow[s, t]$. We denote the conditional expectation with respect to $\mathcal{F}_{t}$ by $\mathbb{E}_{\mathcal{F}_{t}}[\cdot]$ or $\mathbb{E}\left[\cdot \mid \mathcal{F}_{t}\right]$. Under a probability measure $\mathbb{Q}$ different from $\mathbb{P}$, we explicitly denote it, for example, by $\mathbb{E}_{\mathcal{F}_{t}}^{\mathbb{Q}}[\cdot]$. Sometimes we use the abbreviations $\|x\|_{[s, t]}:=\sup _{v \in[s, t]}\left|x_{v}\right|$ and $\Theta_{v}:=\left(Y_{v}, Z_{v}, \psi_{v}\right)$.

We introduce the following spaces. $p \in \mathbb{N}$ is assumed to be $p \geq 2$.

- $\mathbb{D}[s, t]$ is the set of real valued càdlàg functions $\left(q_{v}\right)_{v \in[s, t]}$.

- $\mathbb{S}^{p}[s, t]$ is the set of real (or vector) valued càdlàg $\mathbb{F}$-adapted processes $\left(X_{v}\right)_{v \in[s, t]}$ such that

$$
\|X\|_{\mathbb{S}^{p}[s, t]}:=\mathbb{E}\left[\sup _{v \in[s, t]}\left|X_{v}\right|^{p}\right]^{\frac{1}{p}}<\infty .
$$

- $\mathbb{S}^{\infty}[s, t]$ is the set of real (or vector) valued càdlàg $\mathbb{F}$-adapted processes $\left(X_{v}\right)_{v \in[s, t]}$ which are essentially bounded, i.e.

$$
\|X\|_{\mathbb{S}^{\infty}[s, t]}:=\left\|\sup _{v \in[s, t]}\left|X_{v}\right|\right\|_{\infty}<\infty .
$$

Here, $\|x\|_{\infty}:=\inf \{c \in \mathbb{R} ; \mathbb{P}(\{|x| \leq c\})=1\}$.

- $\mathbb{H}^{p}[s, t]$ is the set of progressively measurable real (or vector) valued processes $\left(Z_{v}\right)_{v \in[s, t]}$ such that

$$
\|Z\|_{\mathbb{H}^{p}[s, t]}:=\mathbb{E}\left[\left(\int_{s}^{t}\left|Z_{v}\right|^{2} d v\right)^{\frac{p}{2}}\right]^{\frac{1}{p}}<\infty .
$$

- $\mathbb{L}^{2}(E, \nu)$ (or simply $\mathbb{L}^{2}(\nu)$ ) is the set of $k$-dimensional vector-valued functions $\psi=\left(\psi^{i}\right)_{1 \leq i \leq k}$ for which the each component $\psi^{i}: \mathbb{R}_{0} \rightarrow \mathbb{R}$ is $\mathcal{B}\left(\mathbb{R}^{0}\right)$-measurable and

$$
\|\psi\|_{\mathbb{L}^{2}(E, \nu)}:=\left(\sum_{i=1}^{k} \int_{\mathbb{R}_{0}}\left|\psi^{i}(e)\right|^{2} \nu^{i}(d e)\right)^{\frac{1}{2}}<\infty .
$$


- $\mathbb{L}^{\infty}(E, \nu)$ (or simply $\mathbb{L}^{\infty}(\nu)$ ) is the set of functions $\psi=\left(\psi^{i}\right)_{1 \leq i \leq k}$ for which the each component $\psi^{i}: \mathbb{R}_{0} \rightarrow \mathbb{R}$ is $\mathcal{B}\left(\mathbb{R}^{0}\right)$-measurable and bounded $\nu^{i}(d e)$-a.e. with the standard essential supremum norm.

- $J^{p}[s, t]$ is the set of functions $\psi=\left(\psi^{i}\right)_{1 \leq i \leq k}$ with $\psi^{i}: \Omega \times[s, t] \times \mathbb{R}_{0} \rightarrow \mathbb{R}$ being $\mathcal{P} \otimes \mathcal{B}\left(\mathbb{R}_{0}\right)$ measurable (or we simply say $\psi$ is $\mathcal{P} \otimes \mathcal{E}$-measurable) and satisfy

$$
\|\psi\|_{\mathbb{J} p[s, t]}:=\mathbb{E}\left[\left(\sum_{i=1}^{k} \int_{s}^{t} \int_{\mathbb{R}_{0}}\left|\psi_{v}^{i}(e)\right|^{2} \nu^{i}(d e) d v\right)^{\frac{p}{2}}\right]^{\frac{1}{p}}<\infty .
$$

- We denote $\mathcal{K}^{p}[s, t]=\mathbb{S}^{p}[s, t] \times \mathbb{H}^{p}[s, t] \times \mathbb{J}^{p}[s, t]$ with the norm

$$
\|(Y, Z, \psi)\|_{\mathcal{K}^{p}[s, t]}:=\|Y\|_{\mathbb{S}^{p}[s, t]}+\|Z\|_{\mathbb{H}^{p}[s, t]}+\|\psi\|_{\mathbb{J} p[s, t]} .
$$

For notational simplicity, hereafter we write

$$
\int_{s}^{t} \int_{E} \psi_{r}(e) \widetilde{\mu}(d r, d e):=\sum_{i=1}^{k} \int_{s}^{t} \int_{\mathbb{R}_{0}} \psi_{r}^{i}(e) \widetilde{\mu}^{i}(d r, d e)
$$

and use similar abbreviations for the integrations with respect to $(\mu, \nu)=\left(\mu^{i}, \nu^{i}\right)_{1 \leq i \leq k}$.

- $\mathbb{J}^{\infty}[s, t]$ is the set of $\mathcal{P} \otimes \mathcal{E}$-measurable functions $\psi=\left(\psi^{i}\right)_{1 \leq i \leq k}$ essentially bounded with respect to the measure $d \mathbb{P} \otimes \nu(d e) \otimes d t$ i.e.

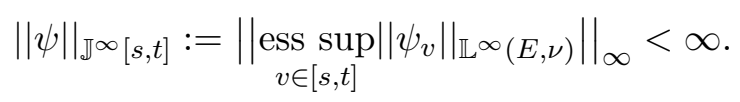

- $\mathbb{H}_{B M O}^{2}[s, t]$ is the set of real (or vector) valued progressively measurable processes $\left(Z_{v}\right)_{v \in[s, t]}$ such that

$$
\|Z\|_{\mathbb{H}_{B M O}^{2}[s, t]}^{2}:=\sup _{\tau \in \mathcal{T}_{s}^{t}}\left\|\mid \mathbb{E}_{\mathcal{F}_{\tau}}\left[\int_{\tau}^{t}\left|Z_{r}\right|^{2} d r\right]\right\|_{\infty}<\infty .
$$

- $\mathbb{J}_{B}^{2}[s, t]$ is the set of $\mathcal{P} \otimes \mathcal{E}$-measurable functions such that

$$
\|\psi\|\left\|_{\mathbb{J}_{B}^{2}[s, t]}^{2}:=\sup _{\tau \in \mathcal{T}_{s}^{t}}\right\| \mathbb{E}_{\mathcal{F}_{\tau}}\left[\int_{\tau}^{t} \int_{E}\left|\psi_{r}(e)\right|^{2} \nu(d e) d r\right] \|_{\infty}<\infty
$$

- $\mathbb{J}_{B M O}^{2}[s, t]$ is the set of $\mathcal{P} \otimes \mathcal{E}$-measurable functions such that

$$
\|\psi\|_{\mathbb{J}_{B M O}^{2}[s, t]}^{2}:=\sup _{\tau \in \mathcal{T}_{s}^{t}}\left\|\mathbb{E}_{\mathcal{F}_{\tau}}\left[\int_{\tau}^{t} \int_{E}\left|\psi_{r}(e)\right|^{2} \nu(d e) d r\right]+\left(\Delta M_{\tau}\right)^{2}\right\|_{\infty}<\infty,
$$

where $\Delta M_{\tau}:=\int_{E} \psi_{\tau}(e) \mu(\{\tau\}, d e)$. See Section 2.3 of [16] and references therein for the details of BMO-martingales with jumps. We frequently omit $[s, t]$ if it is obvious from the context.

\subsection{Some relations among the jump norms $\mathbb{J}^{\infty}, \mathbb{J}_{B}^{2}, \mathbb{J}_{B M O}^{2}$}

By a simple adaptation of Corollary 1 in [26], we get the next Lemma.

Lemma 2.1. Let $\psi$ be in $\mathbb{J}^{2}[0, T]$, and define a square-integrable pure jump martingale 
$\left(M_{t}\right)_{t \in[0, T]}$ by $M_{t}:=\int_{0}^{t} \int_{E} \psi_{s}(e) \widetilde{\mu}(d s, d e)$. The jump $\Delta M$ at time $t$ is given by

$$
\Delta M_{t}:=M_{t}-M_{t-}=\int_{E} \psi_{t}(e) \mu(\{t\}, d e) .
$$

Then the following two conditions are equivalent:

(1) $\|\psi\|_{\mathbb{J} \infty[0, T]}$ is finite.

(2) $\sup _{\tau \in \mathcal{T}_{0}^{T}}\left\|\Delta M_{\tau}\right\|_{\infty}$ is finite.

Moreover, the above two quantities coincide when they exist i.e.

$$
\|\psi\|_{\mathbb{J} \infty}=\sup _{\tau \in \mathcal{T}_{0}^{T}}\left\|\Delta M_{\tau}\right\|_{\infty}
$$

Proof. (1) $\Rightarrow(2)$. Let assume $\psi \in \mathbb{J}^{2}[0, T] \cap \mathbb{J}^{\infty}[0, T]$. By construction, only the jump times of the Poisson measure $\mu(d t, d e)$ contributes $|\Delta M|$. Since the density of $\mu(d t, d e)$ is given by $\nu(d e) \otimes d t$, it is obvious to see $\left|\Delta M_{\tau}\right| \leq\|\psi\|_{J_{\infty}[0, T]}$ a.s. for every stopping time $\tau \in \mathcal{T}_{0}^{T}$ and hence $\sup _{\tau \in \mathcal{T}_{0}^{T}}\left\|\Delta M_{\tau}\right\|_{\infty} \leq\|\psi\|_{\mathbb{J} \infty[0, T]}$.

$(2) \Rightarrow(1)$. Assume conversely, $C:=\sup _{\tau \in \mathcal{T}_{0}^{T}}\left\|\Delta M_{\tau}\right\|_{\infty}<\infty$. By (2.1), one sees

$$
\left|\psi_{\tau}(e)\right| \leq C \quad \text { a.s. }
$$

for every pair $(\tau, e)$ of the jump time $(\tau)$ and its associated mark $(e)$ of the random measure $\mu(d t, d e)$. Let us define a new process $\bar{\psi} \in \mathbb{J}^{2}[0, T] \cap \mathbb{J}^{\infty}[0, T]$ by the next truncation:

$$
\bar{\psi}_{t}(e):=\psi_{t}(e) \mathbf{1}_{\left\{\left|\psi_{t}(e)\right| \leq C\right\}} \quad \forall(\omega, t, e) \in \Omega \times[0, T] \times E .
$$

Notice that $\psi$ and $\bar{\psi}$ are equal a.s. on every jump time and its associated mark of $\mu$. As a consequence, one has

$$
\begin{aligned}
0 & =\mathbb{E}\left[\int_{0}^{T} \int_{E}\left|\psi_{t}(e)-\bar{\psi}_{t}(e)\right|^{2} \mu(d t, d e)\right] \\
& =\mathbb{E}\left[\int_{0}^{T} \int_{E}\left|\psi_{t}(e)-\bar{\psi}_{t}(e)\right|^{2} \nu(d e) d t\right]
\end{aligned}
$$

This means $\psi=\bar{\psi}$ in $\mathbb{J}^{2}[0, T]$ and hence, in particular, $d \mathbb{P} \otimes \nu(d e) \otimes d t$-a.e. Therefore, $\|\psi\|_{\mathbb{J} \infty[0, T]}=\|\bar{\psi}\|_{\mathbb{J}^{\infty}[0, T]} \leq C$ and (1) holds. This establishes the equivalent of (1) and (2). Combining the two results, one can conclude $\|\psi\|_{\mathbb{J} \infty[0, T]}=\sup _{\tau \in \mathcal{T}_{0}^{T}}\left\|\Delta M_{\tau}\right\|_{\infty}$.

Remark 2.1. Note that $\psi$ must be a predictable process. This fact makes the constraint only at the jump points in (2.2) be translated into the whole domain of $\psi$.

Using the above result, one obtains the following relation among the different jump norms.

Lemma 2.2. The following two conditions are equivalent:

(1) $\psi \in \mathbb{J}_{B}^{2}[0, T] \cap \mathbb{J}^{\infty}[0, T]$.

(2) $\psi \in \mathbb{J}_{B M O}^{2}[0, T]$.

Moreover, the following inequality holds:

$$
\left(\|\psi\|_{\mathbb{J}_{B}^{2}[0, T]}^{2} \vee\|\psi\|_{\mathbb{J}^{\infty}[0, T]}^{2}\right) \leq\|\psi\|_{\mathbb{J}_{B M O}^{2}[0, T]}^{2} \leq\|\psi\|_{\mathbb{J}_{B}^{2}[0, T]}^{2}+\|\psi\|_{\mathbb{J} \infty[0, T]}^{2} .
$$

Proof. $(1) \Rightarrow(2)$. Let $\psi$ be in $\mathbb{J}_{B}^{2}[0, T] \cap \mathbb{J}^{\infty}[0, T]$. Since $\mathbb{J}_{B}^{2} \subset \mathbb{J}^{2}$, Lemma 2.1 implies that $\|\psi\|_{\mathbb{J} \infty[0, T]}=\sup _{\tau \in \mathcal{T}_{0}^{T}}\left\|\Delta M_{\tau}\right\|_{\infty}$ where $\Delta M_{\tau}:=\int_{E} \psi_{\tau}(e) \mu(\{\tau\}, d e)$ as defined before. One 
then obtains

$$
\begin{aligned}
\|\psi\|_{\mathbb{J}_{B M O}^{2}}^{2}[0, T] & =\sup _{\tau \in \mathcal{T}_{0}^{T}}\left\|\mathbb{E}_{\mathcal{F}_{\tau}}\left[\int_{\tau}^{T} \int_{E}\left|\psi_{r}(e)\right|^{2} \nu(d e) d r\right]+\left(\Delta M_{\tau}\right)^{2}\right\|_{\infty} \\
& \leq\|\psi\|_{\mathbb{J}_{B}^{2}[0, T]}^{2}+\sup _{\tau \in \mathcal{T}_{0}^{T}}\left\|\Delta M_{\tau}\right\|_{\infty}^{2}=\|\psi\|_{\mathbb{J}_{B}^{2}[0, T]}^{2}+\|\psi\|_{\mathbb{J}_{\infty}[0, T]}^{2} .
\end{aligned}
$$

Thus (2) holds.

$(2) \Rightarrow(1)$. On the other hand let $\psi \in \mathbb{J}_{B M O}^{2}[0, T]$. By definition of $\mathbb{J}_{B M O}^{2}$-norm, one has

$$
\sup _{\tau \in \mathcal{T}_{0}^{T}}\left\|\mathbb{E}_{\mathcal{F}_{\tau}}\left[\int_{\tau}^{T}\left|\psi_{r}(e)\right|^{2} \nu(d e) d r\right]\right\|_{\infty} \vee \sup _{\tau \in \mathcal{T}_{0}^{T}}\left\|\Delta M_{\tau}\right\|_{\infty}^{2} \leq\|\psi\|_{\mathbb{J}_{B M O}^{2}}^{2}
$$

Since $\mathbb{J}_{B M O}^{2} \subset \mathbb{J}_{B}^{2}$, Lemma 2.1 once again implies

$$
\|\psi\|_{\mathbb{J}_{B[0, T]}^{2}}^{2} \vee\|\psi\|_{\mathbb{J}^{\infty}[0, T]}^{2} \leq\|\psi\|_{\mathbb{J}_{B M O}^{2}}^{2}[0, T]
$$

The last claim direct follows from the above two inequalities.

Remark 2.2. When $\psi$ is given as a part of BSDE solution $(Y, Z, \psi)$ as in (3.1), $\psi$ can be defined only up $d \mathbb{P} \otimes \nu(d e) \otimes d t$-a.e. Thus, if one has a $\psi \in \mathbb{J}^{\infty}$, one can freely work on its version $\left(\widetilde{\psi}_{t}(\omega, e)\right)_{(\omega, t, e) \in \Omega \times[0, T] \times E}$ which is everywhere bounded (as in $\bar{\psi}$ used in the proof of Lemma 2.1). This fact is being used in some existing literature.

\section{$3 \quad$ A priori estimates}

\subsection{Universal bounds}

In this section, we consider various a priori estimates regarding anticipated quadratic-exponential growth BSDEs with jumps in a general non-Markovian setup. We are interested in the following ABSDE for $t \in[0, T]$ :

$$
Y_{t}=\xi+\int_{t}^{T} \mathbb{E}_{\mathcal{F}_{r}} f\left(r,\left(Y_{v}\right)_{v \in[r, T]}, Y_{r}, Z_{r}, \psi_{r}\right) d r-\int_{t}^{T} Z_{r} d W_{r}-\int_{t}^{T} \int_{E} \psi_{r}(e) \widetilde{\mu}(d r, d e),
$$

where $f: \Omega \times[0, T] \times \mathbb{D}[0, T] \times \mathbb{R} \times \mathbb{R}^{1 \times d} \times \mathbb{L}^{2}(E, \nu) \rightarrow \mathbb{R}$, and $\xi$ is an $\mathcal{F}_{T}$-measurable random variable.

Assumption 3.1. (i) The driver $f$ is a map such that for every $(y, z, \psi) \in \mathbb{R} \times \mathbb{R}^{1 \times d} \times \mathbb{L}^{2}(E, \nu)$ and any càdlàg $\mathbb{F}$-adapted process $\left(Y_{v}\right)_{v \in[0, T]}$, the process $\left(\mathbb{E}_{\mathcal{F}_{t}} f\left(t,\left(Y_{v}\right)_{v \in[t, T]}, y, z, \psi\right), t \in\right.$ $[0, T])$ is $\mathbb{F}$-progressively measurable, and the map $(y, z, \psi) \rightarrow f(\cdot, y, z, \psi)$ is continuous.

(ii) For every $(q, y, z, \psi) \in \mathbb{D}[0, T] \times \mathbb{R} \times \mathbb{R}^{1 \times d} \times \mathbb{L}^{2}(E, \nu)$, there exist constants $\beta, \delta \geq 0, \gamma>0$ and a positive progressively measurable process $\left(l_{v}, v \in[0, T]\right)$ such that

$$
\begin{aligned}
-l_{t} & -\delta\left(\sup _{v \in[t, T]}\left|q_{v}\right|\right)-\beta|y|-\frac{\gamma}{2}|z|^{2}-\int_{E} j_{\gamma}(-\psi(e)) \nu(d e) \leq f\left(t,\left(q_{v}\right)_{v \in[t, T]}, y, z, \psi\right) \\
& \leq l_{t}+\delta\left(\sup _{v \in[t, T]}\left|q_{v}\right|\right)+\beta|y|+\frac{\gamma}{2}|z|^{2}+\int_{E} j_{\gamma}(\psi(e)) \nu(d e)
\end{aligned}
$$

$d \mathbb{P} \otimes d t$-a.e. $(\omega, t) \in \Omega \times[0, T]$, where $j_{\gamma}(u):=\frac{1}{\gamma}\left(e^{\gamma u}-1-\gamma u\right)$.

(iii) $\|\xi\|_{\infty},\|l\|_{\mathbb{S} \infty}<\infty$. 
Lemma 3.1. Under Assumption [3.1, if there exists a bounded solution $(Y, Z, \psi) \in \mathbb{S}^{\infty} \times \mathbb{H}^{2} \times$ $\mathbb{J}^{2}$ to the $A B S D E$ (3.1), then $Z \in \mathbb{H}_{B M O}^{2}$ and $\psi \in \mathbb{J}_{B M O}^{2}$ (hence $\psi \in \mathbb{J}^{\infty}$ ) and they satisfy

$$
\begin{aligned}
\|Z\|_{\mathbb{H}_{B M O}^{2}}^{2} \leq \frac{e^{4 \gamma\|Y\|_{\mathbb{S} \infty}}}{\gamma^{2}}\left(1+2 \gamma T\left[\|l\|_{\mathbb{S} \infty}+(\beta+\delta)\|Y\|_{\mathbb{S} \infty}\right]\right) \\
\|\psi\|_{\mathbb{J}_{B M O}^{2}}^{2} \leq \frac{e^{4 \gamma\|Y\|_{\mathbb{S} \infty}}}{\gamma^{2}}\left(2+4 \gamma T\left[\|l\|_{\mathbb{S} \infty}+(\beta+\delta)\|Y\|_{\mathbb{S} \infty}\right]\right)+4\|Y\|_{\mathbb{S} \infty}^{2} .
\end{aligned}
$$

Proof. It follows from Lemma 3.1 [16] by a simple replacement of $\|l\|_{\mathbb{S} \infty}$ with $\|l\|_{\mathbb{S} \infty}+\delta\|Y\|_{\mathbb{S} \infty}$. One also needs the fact that $\|\psi\|_{\mathbb{J}_{B M O}^{2}}^{2} \leq\|\psi\|_{\mathbb{J}_{B}^{2}}^{2}+\|\psi\|_{\mathbb{J} \infty}^{2}$ and $\|\psi\|_{\mathbb{J} \infty} \leq 2\|Y\|_{\mathbb{S} \infty}$ from Lemma 2.1. We give details in Appendix B.1.

Lemma 3.2. Under Assumption [3.1, if there exists a bounded solution $(Y, Z, \psi) \in \mathbb{S}^{\infty} \times \mathbb{H}^{2} \times$ $\mathbb{J}^{2}$ to the ABSDE (3.1), then $Y$ has the following estimate

$$
\|Y\|_{\mathbb{S} \infty} \leq \exp \left(T\left(\beta+\delta e^{\beta T}\right)\right)\left(\|\xi\|_{\infty}+T\|l\|_{\mathbb{S} \infty}\right) .
$$

Proof. Applying Mayer-Ito formula, one obtains

$$
d\left(e^{\beta s}\left|Y_{s}\right|\right)=e^{\beta s}\left(\beta\left|Y_{s}\right| d s+\operatorname{sign}\left(Y_{s-}\right) d Y_{s}+d L_{s}\right) .
$$

Here, $\left(L_{s}\right)_{s \in[0, T]}$ is a non-decreasing process including a local time $L^{c}$ as

$$
d L_{s}=d L_{s}^{c}+\int_{E}\left(\left|Y_{s-}+\psi_{s}(e)\right|-\left|Y_{s-}\right|-\operatorname{sign}\left(Y_{s-}\right) \psi_{s}(e)\right) \mu(d s, d e) .
$$

Note that

$$
|y+\psi|-|y|-\operatorname{sign}(y) \psi=|y+\psi|-\operatorname{sign}(y)(y+\psi) \geq 0 .
$$

Let us introduce the following processes $\left(B_{s}\right)_{s \in[0, T]}$ and $\left(C_{s}\right)_{s \in[0, T]}$ by

$$
\begin{aligned}
d B_{s}= & -\operatorname{sign}\left(Y_{s}\right) \mathbb{E}_{\mathcal{F}_{s}} f\left(s,\left(Y_{v}\right)_{v \in[s, T]}, \Theta_{s}\right) d s \\
& +\left(l_{s}+\delta \mathbb{E}_{\mathcal{F}_{s}}\left(\sup _{v \in[s, T]}\left|Y_{v}\right|\right)+\beta\left|Y_{s}\right|+\frac{\gamma}{2}\left|Z_{s}\right|^{2}+\int_{E} j_{\gamma}\left(\operatorname{sign}\left(Y_{s}\right) \psi_{s}(e)\right) \nu(d e)\right) d s, \\
d C_{s}= & e^{\beta s}\left(d B_{s}+d L_{s}\right)+\frac{\gamma}{2}\left(e^{2 \beta s}-e^{\beta s}\right)\left|Z_{s}\right|^{2} d s \\
& +\int_{E}\left(j_{\gamma}\left(e^{\beta s} \operatorname{sign}\left(Y_{s}\right) \psi_{s}(e)\right)-e^{\beta s} j_{\gamma}\left(\operatorname{sign}\left(Y_{s}\right) \psi_{s}(e)\right)\right) \nu(d e) d s .
\end{aligned}
$$

Note that both of $B$ and $C$ are non-decreasing processes. As for the process $B$, this follows from Assumption 3.1. As for the process $C$, it follows from the fact that $k \geq 1, j_{\gamma}(k u)-$ $k j_{\gamma}(u)=\frac{1}{\gamma}\left(e^{k \gamma u}-k e^{\gamma u}-1+k\right) \geq 0$, which makes the last line positive. One then sees

$$
\begin{gathered}
d\left(e^{\beta s}\left|Y_{s}\right|+\int_{0}^{s} e^{\beta r}\left(l_{r}+\delta \mathbb{E}_{\mathcal{F}_{r}}\left(\sup _{v \in[r, T]}\left|Y_{v}\right|\right)\right) d r\right)=e^{\beta s} \operatorname{sign}\left(Y_{s-}\right)\left(Z_{s} d W_{s}+\int_{E} \psi_{s}(e) \widetilde{\mu}(d s, d e)\right) \\
-\int_{E} j_{\gamma}\left(e^{\beta s} \operatorname{sign}\left(Y_{s}\right) \psi_{s}(e)\right) \nu(d e) d s-\frac{\gamma}{2}\left|e^{\beta s} \operatorname{sign}\left(Y_{s}\right) Z_{s}\right|^{2} d s+d C_{s} .
\end{gathered}
$$


We now investigate the process $P_{t}, t \in[0, T]$ defined by

$$
P_{t}:=\exp \left(\gamma e^{\beta t}\left|Y_{t}\right|+\gamma \int_{0}^{t} e^{\beta r}\left(l_{r}+\delta \mathbb{E}_{\mathcal{F}_{r}}\left(\sup _{v \in[r, T]}\left|Y_{v}\right|\right)\right) d r\right), \quad t \in[0, T],
$$

where $P \in \mathbb{S}^{\infty}$ is clearly seen. Applying Ito formula, one obtains that

$$
\begin{aligned}
d P_{t} & =P_{t-} \gamma d\left(e^{\beta t}\left|Y_{t}\right|+\int_{0}^{t} e^{\beta r}\left(l_{r}+\delta \mathbb{E}_{\mathcal{F}_{r}} \sup _{v \in[r, T]}\left|Y_{v}\right|\right) d r\right)+P_{t} \frac{\gamma^{2}}{2}\left|e^{\beta t} \operatorname{sign}\left(Y_{t}\right) Z_{t}\right|^{2} d t \\
& +P_{t-} \int_{E}\left(e^{\gamma e^{\beta t}\left(\left|Y_{t-}+\psi_{t}(e)\right|-\mid Y_{t-\mid}\right)}-1-\gamma e^{\beta t} \operatorname{sign}\left(Y_{t-}\right) \psi_{t}(e)\right) \mu(d t, d e) \\
= & P_{t-}\left(\gamma e^{\beta t} \operatorname{sign}\left(Y_{t}\right) Z_{t} d W_{t}+\int_{E}\left(\exp \left(\gamma e^{\beta t} \operatorname{sign}\left(Y_{t-}\right) \psi_{t}(e)\right)-1\right) \widetilde{\mu}(d t, d e)+d C_{t}^{\prime}\right)
\end{aligned}
$$

where $\left(C_{s}^{\prime}\right)_{s \in[0, T]}$ is another non-decreasing (see $(\underline{3.2})$ ) process defined by

$$
d C_{t}^{\prime}=\gamma d C_{t}+\int_{E}\left(e^{\gamma e^{\beta t}\left(\left|Y_{t-}+\psi_{t}(e)\right|-\left|Y_{t-}\right|\right)}-e^{\gamma e^{\beta t} \operatorname{sign}\left(Y_{t-}\right) \psi_{t}(e)}\right) \mu(d t, d e) .
$$

The details of the derivation of (3.4) are given in Appendix B.2.

Since $(P, Y, Z, \psi) \in \mathbb{S}^{\infty} \times \mathbb{S}^{\infty} \times \mathbb{H}_{B M O}^{2} \times \mathbb{J}_{B M O}^{2}$, one sees the process $P$ is a true submartingale. Therefore, it follows that, for any $t \in[0, T]$,

$$
\begin{aligned}
\exp \left(\gamma e^{\beta t}\left|Y_{t}\right|\right) & \leq \mathbb{E}_{\mathcal{F}_{t}}\left[\exp \left(\gamma e^{\beta T}|\xi|+\gamma \int_{t}^{T} e^{\beta r}\left(l_{r}+\delta \mathbb{E}_{\mathcal{F}_{r}}\left(\sup _{v \in[r, T]}\left|Y_{v}\right|\right)\right) d r\right)\right] \\
& \leq \exp \left(\gamma e^{\beta T}\left(\|\xi\|_{\infty}+T\|l\|_{\mathbb{S} \infty}\right)+\gamma \delta e^{\beta T} \int_{t}^{T}\|Y\|_{\mathbb{S} \infty[r, T]} d r\right) \text { a.s. }
\end{aligned}
$$

Thus, $\left|Y_{t}\right| \leq e^{\beta T}\left(\|\xi\|_{\infty}+T\|l\|_{\mathbb{S} \infty}\right)+\delta e^{\beta T} \int_{t}^{T}\|Y\|_{\mathbb{S} \infty[r, T]} d r$ a.s. Since the right-hand side is non-increasing in $t$, the same inequality holds with the left-hand side replaced by $\sup _{s \in[t, T]}\left|Y_{s}\right|$. Hence equivalently,

$$
\|Y\|_{\mathbb{S}^{\infty}[t, T]} \leq e^{\beta T}\left(\|\xi\|_{\infty}+T\|l\|_{\mathbb{S} \infty}\right)+\delta e^{\beta T} \int_{t}^{T}\|Y\|_{\mathbb{S}^{\infty}[r, T]} d r
$$

Now using the backward Gronwall inequality 1, one obtains the desired result.

Definition 3.1. We define the set of parameters $A:=\left(\|\xi\|_{\infty},\|l\|_{\mathbb{S} \infty}, \delta, \beta, \gamma, T\right)$ which control the universal bounds on $\left(\|Y\|_{\mathbb{S} \infty},\|Z\|_{\mathbb{H}_{B M O}^{2}},\|\psi\|_{\mathbb{J}_{B M O}^{2}}\right)$.

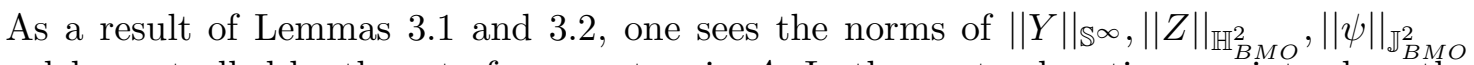
are solely controlled by the set of parameters in $A$. In the next subsection, we introduce the local Lipschitz continuity.

\subsection{Stability and Uniqueness}

Assumption 3.2. For each $M>0$, and for every $(q, y, z, \psi),\left(q^{\prime}, y^{\prime}, z^{\prime}, \psi^{\prime}\right) \in \mathbb{D}[0, T] \times \mathbb{R} \times$ $\mathbb{R}^{1 \times d} \times \mathbb{L}^{2}(E, \nu)$ satisfying $\sup _{v \in[0, T]}\left|q_{v}\right|, \sup _{v \in[0, T]}\left|q_{v}^{\prime}\right|,|y|,\left|y^{\prime}\right|,\|\psi\|_{\mathbb{L}^{\infty}(\nu)},\left\|\psi^{\prime}\right\|_{\mathbb{L}^{\infty}(\nu)} \leq M$,

\footnotetext{
${ }^{1}$ See, for example, Corollary 6.61 31]
} 
there exists some positive constant $K_{M}$ (depending on $M$ ) such that

$$
\begin{aligned}
& \left|f\left(t,\left(q_{v}\right)_{v \in[t, T]}, y, z, \psi\right)-f\left(t,\left(q_{v}^{\prime}\right)_{v \in[t, T]}, y^{\prime}, z^{\prime}, \psi^{\prime}\right)\right| \\
& \quad \leq K_{M}\left(\sup _{v \in[t, T]}\left|q_{v}-q_{v}^{\prime}\right|+\left|y-y^{\prime}\right|+|| \psi-\psi^{\prime} \|_{\mathbb{L}^{2}(\nu)}\right) \\
& \quad+K_{M}\left(1+|z|+\left|z^{\prime}\right|+|| \psi \|_{\mathbb{L}^{2}(\nu)}+\left.|| \psi^{\prime}\right|_{\mathbb{L}^{2}(\nu)}\right)\left|z-z^{\prime}\right|
\end{aligned}
$$

$d \mathbb{P} \otimes d t$-a.e. $(\omega, t) \in \Omega \times[0, T]$.

Remark 3.1. Instead of directly making the driver $f$ path-dependent, one can include the conditional expectations such as $\mathbb{E}_{\mathcal{F}_{t}}\left(Y_{t+\delta}\right), \mathbb{E}_{\mathcal{F}_{t}}\left(\int_{t}^{T} Y_{s} d s\right)$ as done in [28]. In this work, we adopt the former approach since it allows the general dependence without specifying a concrete form.

Let us introduce the two ABSDEs for $t \in[0, T]$, with $i=\{1,2\}$,

$$
Y_{t}^{i}=\xi^{i}+\int_{t}^{T} \mathbb{E}_{\mathcal{F}_{r}} f^{i}\left(r,\left(Y_{v}^{i}\right)_{v \in[r, T]}, Y_{r}^{i}, Z_{r}^{i}, \psi_{r}^{i}\right) d r-\int_{t}^{T} Z_{r}^{i} d W_{r}-\int_{t}^{T} \int_{E} \psi_{r}^{i}(e) \widetilde{\mu}(d r, d e) .
$$

Let us put $\delta Y:=Y^{1}-Y^{2}, \delta Z:=Z^{1}-Z^{2}, \delta \psi:=\psi^{1}-\psi^{2}$, and

$$
\delta f(r):=\left(f^{1}-f^{2}\right)\left(r,\left(Y_{v}^{1}\right)_{v \in[r, T]}, Y_{r}^{1}, Z_{r}^{1}, \psi_{r}^{1}\right) .
$$

Then, we have the following stability result.

Proposition 3.1. Suppose that the data $\left(\xi^{i}, f^{i}\right)_{1 \leq i \leq 2}$ satisfy Assumptions 3.1 and 3.2. If the two ABSDEs (3.7) have bounded solutions $\left(Y^{i}, Z^{i}, \psi^{i}\right)_{1 \leq i \leq 2} \in \mathbb{S}^{\infty} \times \mathbb{H}^{2} \times \mathbb{J}^{2}$, then for any $p>2 q_{*}$

$$
\|\delta Y\|_{\mathbb{S}^{p}[0, T]} \leq C_{1} \mathbb{E}\left[|\delta \xi|^{p}+\left(\int_{0}^{T} \mathbb{E}_{\mathcal{F}_{r}}|\delta f(r)| d r\right)^{p}\right]^{\frac{1}{p}}
$$

and for any $p \geq 2, \bar{q} \geq q_{*}$

$$
\|\left.(\delta Y, \delta Z, \delta \psi)\right|_{\mathcal{K}^{p}[0, T]} \leq C_{2} \mathbb{E}\left[|\delta \xi|^{\bar{q}^{2}}+\left(\int_{0}^{T} \mathbb{E}_{\mathcal{F}_{r}}|\delta f(r)| d r\right)^{p \bar{q}^{2}}\right]^{\frac{1}{p \bar{q}^{2}}}
$$

where $q_{*} \in(1, \infty)$ is a constant depending only on $(K, A), C_{1}=C(p, K ., A)$ and $C_{2}=$ $(p, \bar{q}, K ., A)$ are two positive constants.

Proof. Note that one can apply (3.6) globally with fixed $K_{M}$ by choosing $M$ larger than the bounds implied from Lemmas 3.1 and 3.2. Let fix such an $M$ in the remainder. Define the $\mathbb{R}^{d}$-valued progressively measurable process $\left(b_{r}, r \in[0, T]\right)$ by

$$
b_{r}:=\frac{\mathbb{E}_{\mathcal{F}_{r}}\left[f^{2}\left(r,\left(Y_{v}^{1}\right)_{v \in[r, T]}, Y_{r}^{1}, Z_{r}^{1}, \psi_{r}^{1}\right)-f^{2}\left(r,\left(Y_{v}^{1}\right)_{v \in[r, T]}, Y_{r}^{1}, Z_{r}^{2}, \psi_{r}^{1}\right)\right]}{\left|\delta Z_{r}\right|^{2}} \mathbf{1}_{\delta Z_{r} \neq 0} \delta Z_{r}^{\top} .
$$

Since $\left|b_{r}\right| \leq K_{M}\left(1+\left|Z_{r}^{1}\right|+\left|Z_{r}^{2}\right|+2|| \psi_{r}^{1} \|_{\mathbb{L}^{2}(\nu)}^{2}\right)$, there exists some constant $C$ such that $\|b\|_{\mathbb{H}_{B M O}^{2}} \leq C$ with $C=C(K, A)$. Thus one can define an equivalent probability measure $\mathbb{Q}$ by $\frac{d \mathbb{Q}}{d \mathbb{P}}=\mathcal{E}_{T}\left(\int_{0}^{\cdot} b_{r}^{\top} d W_{r}\right)$ where $\mathcal{E}(\cdot)$ is Doléans-Dade exponential. We have $W^{\mathbb{Q}}=W-\int_{0} b_{r} d r$ and the Poisson measure is unchanged, $\widetilde{\mu}^{\mathbb{Q}}=\widetilde{\mu}$. We also have $\frac{d \mathbb{P}}{d \mathbb{Q}}=\mathcal{E}_{T}\left(-\int_{0}^{\cdot} b_{r}^{\top} d W_{r}^{\mathbb{Q}}\right)$. From Remark A.1, there exists some constant $r^{*} \in(1, \infty)$ such that the reverse Hölder inequality holds for both of the $\mathcal{E}$. $\left(\int_{0}^{\cdot} b_{r}^{\top} d W_{r}\right)$ and $\mathcal{E}$. $\left(-\int_{0}^{\cdot} b_{r}^{\top} d W_{r}^{\mathbb{Q}}\right)$ with power $\bar{r} \in\left(1, r^{*}\right]$. Define $q_{*}>1$ by $q_{*}:=r^{*} /\left(r^{*}-1\right)$. Note that $\left(r^{*}, q_{*}\right)$ are solely controlled by $(K, A)$. 
Under the measure $\mathbb{Q}$, we have

$$
\begin{aligned}
\delta Y_{t} & =\delta \xi+\int_{t}^{T} \mathbb{E}_{\mathcal{F}_{r}}\left[\delta f(r)+f^{2}\left(r,\left(Y_{v}^{1}\right)_{v \in[r, T]}, Y_{r}^{1}, Z_{r}^{2}, \psi_{r}^{1}\right)-f^{2}\left(r,\left(Y_{v}^{2}\right)_{v \in[r, T]}, Y_{r}^{2}, Z_{r}^{2}, \psi_{r}^{2}\right)\right] d r \\
& -\int_{t}^{T} \delta Z_{r} d W_{r}^{\mathbb{Q}}-\int_{t}^{T} \int_{E} \delta \psi_{r}(e) \widetilde{\mu}^{\mathbb{Q}}(d r, d e), t \in[0, T] .
\end{aligned}
$$

[Stability for Y] Applying Ito formula to $\delta Y^{2}$, one obtains

$$
\begin{aligned}
& \left|\delta Y_{t}\right|^{2}+\int_{t}^{T}\left|\delta Z_{r}\right|^{2} d r+\int_{t}^{T} \int_{E}\left|\delta \psi_{r}(e)\right|^{2} \mu(d r, d e) \\
& =|\delta \xi|^{2}+\int_{t}^{T} 2 \delta Y_{r} \mathbb{E}_{\mathcal{F}_{r}}\left[\delta f(r)+f^{2}\left(r,\left(Y_{v}^{1}\right)_{v \in[r, T]}, Y_{r}^{1}, Z_{r}^{2}, \psi_{r}^{1}\right)-f^{2}\left(r,\left(Y_{v}^{2}\right)_{v \in[r, T]}, Y_{r}^{2}, Z_{r}^{2}, \psi_{r}^{2}\right)\right] d r \\
& \quad-\int_{t}^{T} 2 \delta Y_{r} \delta Z_{r} d W_{r}^{\mathbb{Q}}-\int_{t}^{T} \int_{E} 2 \delta Y_{r-} \delta \psi_{r}(e) \widetilde{\mu}^{\mathbb{Q}}(d r, d e) .
\end{aligned}
$$

The last two terms are true $\mathbb{Q}$-martingales, which can be checked by reverse Hölder and energy inequalities. By taking conditional expectation $\mathbb{E}_{\mathcal{F}_{t}}^{\mathbb{Q}}[\cdot]$, one obtains with any $\lambda>0$

$$
\begin{aligned}
\left|\delta Y_{t}\right|^{2} & +\mathbb{E}_{\mathcal{F}_{t}}^{\mathbb{Q}} \int_{t}^{T}\left|Z_{r}\right|^{2} d r+\mathbb{E}_{\mathcal{F}_{t}}^{\mathbb{Q}} \int_{t}^{T}\left\|\delta \psi_{r}\right\|_{\mathbb{L}^{2}(\nu)}^{2} d r \leq C \mathbb{E}_{\mathcal{F}_{t}}^{\mathbb{Q}} \int_{t}^{T} \mathbb{E}_{\mathcal{F}_{r}}\left[\|\delta Y\|_{[r, T]}^{2}\right] d r \\
& +\mathbb{E}_{\mathcal{F}_{t}}^{\mathbb{Q}}\left[|\delta \xi|^{2}+\frac{1}{\lambda}\left(\int_{t}^{T} \mathbb{E}_{\mathcal{F}_{r}}|\delta f(r)| d r\right)^{2}+\lambda\|\delta Y\|_{[t, T]}^{2}\right]+\frac{1}{2} \mathbb{E}_{\mathcal{F}_{t}}^{\mathbb{Q}} \int_{t}^{T}\left\|\delta \psi_{r}\right\|_{\mathbb{L}^{2}(\nu)}^{2} d r
\end{aligned}
$$

with some positive constant $C=C(K, A)$. Here we have used the fact that $\left|\delta Y_{r}\right| \leq$ $\mathbb{E}_{\mathcal{F}_{r}}\left[\|\delta Y\|_{[r, T]}\right]$. Therefore, in particular,

$$
\begin{aligned}
& \left|\delta Y_{t}\right|^{2} \leq \mathbb{E}_{\mathcal{F}_{t}}^{\mathbb{Q}}\left[|\delta \xi|^{2}+\frac{1}{\lambda}\left(\int_{t}^{T} \mathbb{E}_{\mathcal{F}_{r}}|\delta f(r)| d r\right)^{2}+\lambda\|\delta Y\|_{[t, T]}^{2}+C \int_{t}^{T} \mathbb{E}_{\mathcal{F}_{r}}\left[\|\delta Y\|_{[r, T]}^{2}\right] d r\right] \\
& =\frac{1}{\mathcal{E}_{t}} \mathbb{E}_{\mathcal{F}_{t}}\left[\mathcal{E}_{T}\left(|\delta \xi|^{2}+\frac{1}{\lambda}\left(\int_{t}^{T} \mathbb{E}_{\mathcal{F}_{r}}|\delta f(r)| d r\right)^{2}+\lambda\|\delta Y\|_{[t, T]}^{2}+C \int_{t}^{T} \mathbb{E}_{\mathcal{F}_{r}}\left[\|\delta Y\|_{[r, T]}^{2}\right] d r\right)\right]
\end{aligned}
$$

where $\mathcal{E}_{s}:=\mathcal{E}_{s}\left(\int_{0}^{\cdot} b_{r}^{\top} d W_{r}\right)$. Choosing $\bar{q} \in\left[q_{*}, \infty\right)$, the reverse Hölder inequality yields

$$
\begin{aligned}
& \left|\delta Y_{t}\right|^{2 \bar{q}} \leq C \mathbb{E}_{\mathcal{F}_{t}}\left[|\delta \xi|^{2 \bar{q}}+\frac{1}{\lambda^{\bar{q}}}\left(\int_{t}^{T} \mathbb{E}_{\mathcal{F}_{r}}|\delta f(r)| d r\right)^{2 \bar{q}}+\left(\int_{t}^{T} \mathbb{E}_{\mathcal{F}_{r}}\left[\|\delta Y\|_{[r, T]}^{2}\right] d r\right)^{\bar{q}}+\lambda^{\bar{q}}\|\delta Y\|_{[t, T]}^{2 \bar{q}}\right] \\
& \leq C \mathbb{E}_{\mathcal{F}_{t}}\left[|\delta \xi|^{2 \bar{q}}+\frac{1}{\lambda^{\bar{q}}}\left(\int_{t}^{T} \mathbb{E}_{\mathcal{F}_{r}}|\delta f(r)| d r\right)^{2 \bar{q}}+\int_{t}^{T}\|\delta Y\|_{[r, T]}^{2 \bar{q}} d r+\lambda^{\bar{q}}\|\delta Y\|_{[t, T]}^{2 \bar{q}}\right]
\end{aligned}
$$

with some $C=C(\bar{q}, K ., A)$, where in the 2 nd line Jensen's inequality was used. For any $p>2 \bar{q}$, applying Doob's maximal inequality, one obtains

$\mathbb{E}\left[\|\delta Y\|_{[s, T]}^{p}\right] \leq C \mathbb{E}\left[|\delta \xi|^{p}+\frac{1}{\lambda^{\frac{p}{2}}}\left(\int_{s}^{T} \mathbb{E}_{\mathcal{F}_{r}}|\delta f(r)| d r\right)^{p}\right]+C \int_{s}^{T} \mathbb{E}\left[\|\delta Y\|_{[r, T]}^{p}\right] d r+C \lambda^{\frac{p}{2}} \mathbb{E}\left[\|\delta Y\|_{[s, T]}^{p}\right]$

with $C=C(p, \bar{q}, K, A)$. Choosing $\lambda>0$ small enough so that $C \lambda^{\frac{p}{2}}<1$, the backward Gronwall inequality implies

$$
\mathbb{E}\left[\sup _{t \in[s, T]}\left|\delta Y_{t}\right|^{p}\right] \leq C \mathbb{E}\left[|\delta \xi|^{p}+\left(\int_{s}^{T} \mathbb{E}_{\mathcal{F}_{r}}|\delta f(r)| d r\right)^{p}\right], \quad \forall s \in[0, T] .
$$


One sees the last inequality holds for any $p>2 q_{*}$. This proves (3.8). Since $1<q_{*} \leq \bar{q}$, it also follows that

$$
\mathbb{E}\left[\sup _{t \in[0, T]}\left|\delta Y_{t}\right|^{p}\right]^{\frac{1}{p}} \leq \mathbb{E}\left[\sup _{t \in[0, T]}\left|\delta Y_{t}\right|^{p \bar{q}^{2}}\right]^{\frac{1}{p \bar{q}^{2}}} \leq C \mathbb{E}\left[|\delta \xi|^{p \bar{q}^{2}}+\left(\int_{0}^{T} \mathbb{E}_{\mathcal{F}_{r}}|\delta f(r)| d r\right)^{p \bar{q}^{2}}\right]^{\frac{1}{p \bar{q}^{2}}}
$$

with $C=C(p, \bar{q}, K ., A)$ for any $p \geq 2$.

[Stability for $Z$ and $\psi$ ] From (3.10), one has with $C=C(K ., A)$,

$$
\begin{aligned}
& \left|\delta Y_{t}\right|^{2}+\int_{t}^{T}\left|\delta Z_{r}\right|^{2} d r+\int_{t}^{T} \int_{E}\left|\delta \psi_{r}(e)\right|^{2} \mu(d r, d e) \\
& \leq|\delta \xi|^{2}+\left(\int_{t}^{T} \mathbb{E}_{\mathcal{F}_{r}}|\delta f(r)| d r\right)^{2}+\|\delta Y\|_{[t, T]}^{2}+C \int_{t}^{T} \mathbb{E}_{\mathcal{F}_{r}}\left[\|\delta Y\|_{[r, T]}^{2}\right] d r \\
& \quad+C \int_{t}^{T}\left|\delta Y_{r}\right|\left\|\delta \psi_{r}\right\|_{\mathbb{L}^{2}(\nu)} d r-\int_{t}^{T} 2 \delta Y_{r} \delta Z_{r} d W_{r}^{\mathbb{Q}}-\int_{t}^{T} \int_{E} 2 \delta Y_{r-} \delta \psi_{r}(e) \widetilde{\mu}^{\mathbb{Q}}(d r, d e) .
\end{aligned}
$$

For any $p \geq 2$, applying Burkholder-Davis-Gundy inequality and Lemma A.3, one can show that there exists some constant $C=C(p, K$., A) such that

$$
\begin{aligned}
& \mathbb{E}^{\mathbb{Q}}\left[\left(\int_{0}^{T}\left|\delta Z_{r}\right|^{2} d r\right)^{\frac{p}{2}}\right]+\mathbb{E}^{\mathbb{Q}}\left[\left(\int_{0}^{T} \int_{E}\left|\delta \psi_{r}(e)\right|^{2} \mu(d r, d e)\right)^{\frac{p}{2}}\right] \\
& \leq C \mathbb{E}^{\mathbb{Q}}\left[|\delta \xi|^{p}+\left(\int_{0}^{T} \mathbb{E}_{\mathcal{F}_{r}}|\delta f(r)| d r\right)^{p}+\sup _{r \in[0, T]} \mathbb{E}_{\mathcal{F}_{r}}\left[\|\delta Y\|_{[0, T]}^{p}\right]+\|\delta Y\|_{[0, T]}^{p}\right] .
\end{aligned}
$$

Taking $\bar{q} \geq q_{*}$, the reverse Hölder and Doob's maximal inequalities give

$$
\begin{aligned}
& \mathbb{E}^{\mathbb{Q}}\left[\left(\int_{0}^{T}\left|\delta Z_{r}\right|^{2} d r\right)^{\frac{p}{2}}\right]^{\frac{1}{p}}+\mathbb{E}^{\mathbb{Q}}\left[\left(\int_{0}^{T} \int_{E}\left|\delta \psi_{r}(e)\right|^{2} \mu(d r, d e)\right)^{\frac{p}{2}}\right]^{\frac{1}{p}} \\
& \leq C \mathbb{E}\left[|\delta \xi|^{p \bar{q}}+\left(\int_{0}^{T} \mathbb{E}_{\mathcal{F}_{r}}|\delta f(r)| d r\right)^{p \bar{q}}+\sup _{r \in[0, T]} \mathbb{E}_{\mathcal{F}_{r}}\left[\|\delta Y\|_{[0, T]}^{p}\right]^{\bar{q}}+\|\delta Y\|_{[0, T]}^{p \bar{q}}\right]^{\frac{1}{p \bar{q}}} \\
& \leq C \mathbb{E}\left[|\delta \xi|^{p \bar{q}}+\left(\int_{0}^{T} \mathbb{E}_{\mathcal{F}_{r}}|\delta f(r)| d r\right)^{p \bar{q}}+\|\delta Y\|_{[0, T]}^{p \bar{q}}\right]^{\frac{1}{p \bar{q}}}
\end{aligned}
$$

The reverse Hölder inequality implies $\|Z\|_{\mathbb{H}^{p}}+\|\psi\|_{\mathbb{J}^{P}} \leq C\left(\|Z\|_{\mathbb{H}^{p \bar{q}}(\mathbb{Q})}+\|\psi\|_{J^{p \bar{q}}(\mathbb{Q})}\right)$. Thus the estimate of (3.11) and Lemma A.3 give

$$
\|\delta Y\|_{\mathbb{S}^{p}}+\|\delta Z\|_{\mathbb{H}^{p}}+\|\delta \psi\|_{\mathbb{J}^{p}} \leq C \mathbb{E}\left[|\delta \xi|^{p \bar{q}^{2}}+\left(\int_{0}^{T} \mathbb{E}_{\mathcal{F}_{r}}|\delta f(r)| d r\right)^{p \bar{q}^{2}}\right]^{\frac{1}{p \bar{q}^{2}}}
$$

for any $p \geq 2$ and $\bar{q} \geq q_{*}$ with some positive constant $C=C(p, \bar{q}, K ., A)$.

We also have the following relation.

Lemma 3.3. Under the same conditions used in Proposition 3.1, one has

$$
\|\delta Z\|_{\mathbb{H}_{B M O}^{2}}+\|\delta \psi\|_{\mathbb{J}_{B M O}^{2}} \leq C\left(\|\delta Y\|_{\mathbb{S}^{\infty}}+\|\delta \xi\|_{\infty}+\sup _{t \in \mathcal{T}_{0}^{T}}\left\|\mathbb{E}_{\mathcal{F}_{t}} \int_{t}^{T}|\delta f(r)| d r\right\|_{\infty}\right)
$$

with some positive constant $C=C(K ., A)$.

\footnotetext{
${ }^{2}$ See, for example, Theorem 48 in IV.4. of 33 .
} 
Proof. It follows from a simple modification of Lemma 3.3 (a) of [16].

Combining the results in this section, we obtain the uniqueness.

Corollary 3.1. Under Assumptions 3.1] and [3.2, if the ABSDE (3.1) has a bounded solution $(Y, Z, \psi) \in \mathbb{S}^{\infty} \times \mathbb{H}^{2} \times \mathbb{J}^{2}$, then it is unique with respect to the norm $\mathbb{S}^{\infty} \times \mathbb{H}_{B M O}^{2} \times \mathbb{J}_{B M O}^{2}$.

Proof. Proposition 3.1 implies the uniqueness of $Y$ in $\mathbb{S}^{p}, \forall p \geq 2$, in particular. This also implies the uniqueness with respect to $\mathbb{S}^{\infty}$. If not, there exists some $c>0$ such that $\|\delta Y\|_{\mathbb{S} \infty}=$ $c$, which implies for any $0<b<c$, there exists a strictly positive constant $a>0$ such that $\mathbb{P}\left(\sup _{t \in[0, T]}\left|\delta Y_{t}\right|>b\right)=a$. This yields $\|\delta Y\|_{\mathbb{S}^{p}}^{p}>b^{p} a>0$, which is a contradiction. Thus the assertion follows from Proposition 3.1 and Lemma 3.3 .

Remark 3.2. For quadratic BSDEs, allowing the anticipated components of $(Z, \psi)$ in the driver $f$ seems very hard. In fact, we cannot derive the stability result similar to Proposition 3.1. This is because that the use of the reverse Hölder inequality makes the power of $(|Z|,|\psi|)$ different in the left and right hand sides in the relevant inequalities after aligning the probability measure of the conditional expectations to a single one. The anticipated component for $Y$ is an exceptional case, where we can remove one conditional expectation by the simple fact $Y_{t}=\mathbb{E}^{\mathbb{Q}}\left[Y_{t} \mid \mathcal{F}_{t}\right]$. Note that the Proposition 3.1 is necessary also for the non-Markovian settings in Section $\mathbf{6}$. In the absence of the stability result, the convergence using the monotone sequence would be the last hope. However, to the best of our knowledge, no comparison principle is known in the presence of anticipated components of the control variables $(Z, \psi)$.

\section{Existence in a Markovian Setup}

Let us now provide the existence result for a Markovian setting. We introduce the following forward process, for $s \in[0, T]$,

$$
X_{s}^{t, x}=x+\int_{t}^{s \vee t} b\left(r, X_{r}^{t, x}\right) d r+\int_{t}^{s \vee t} \sigma\left(r, X_{r}^{t, x}\right) d W_{r}+\int_{t}^{s \vee t} \int_{E} \gamma\left(r, X_{r-}^{t, x}, e\right) \widetilde{\mu}(d r, d e)
$$

where $x \in \mathbb{R}^{n}$ and $b:[0, T] \times \mathbb{R}^{n} \rightarrow \mathbb{R}^{n}, \sigma:[0, T] \times \mathbb{R}^{n} \rightarrow \mathbb{R}^{n \times d}, \gamma:[0, T] \times \mathbb{R}^{n} \times E \rightarrow \mathbb{R}^{n \times k}$ are non-random measurable functions. Note that $X_{s}^{t, x} \equiv x$ for $s \leq t$.

Assumption 4.1. There exists a positive constant $K$ such that

(i) $|b(t, 0)|+|\sigma(t, 0)| \leq K$ uniformly in $t \in[0, T]$.

(ii) $\sum_{i=1}^{k}\left|\gamma^{i}(t, 0, e)\right| \leq K(1 \wedge|e|)$ uniformly in $(t, e) \in[0, T] \times \mathbb{R}_{0}$.

(iii) uniformly in $t \in[0, T], x, x^{\prime} \in \mathbb{R}^{n}, e \in \mathbb{R}_{0}$,

$$
\begin{aligned}
& \left|b(t, x)-b\left(t, x^{\prime}\right)\right|+\left|\sigma(t, x)-\sigma\left(t, x^{\prime}\right)\right| \leq K\left|x-x^{\prime}\right|, \\
& \sum_{i=1}^{k}\left|\gamma^{i}(t, x, e)-\gamma^{i}\left(t, x^{\prime}, e\right)\right| \leq K(1 \wedge|e|)\left|x-x^{\prime}\right| .
\end{aligned}
$$

Lemma 4.1. Under Assumption 4.1, there exists a unique solution to (4.1) for each $(t, x)$ 
which satisfies for any $(t, x),\left(t, x^{\prime}\right) \in[0, T] \times \mathbb{R}^{n}$ and $p \geq 2$,

(a) $\mathbb{E}\left[\sup _{s \in[0, T]}\left|X_{s}^{t, x}\right|^{p}\right] \leq C\left(1+|x|^{p}\right)$

(b) $\mathbb{E}\left[\sup _{s \leq u \leq(s+h) \wedge T}\left|X_{s}^{t, x}-X_{u}^{t, x}\right|^{p}\right] \leq C\left(1+|x|^{p}\right) h, \forall s \in[0, T]$

(c) $\mathbb{E}\left[\sup _{s \in[0, T]}\left|X_{s}^{t, x}-X_{s}^{t^{\prime}, x^{\prime}}\right|^{p}\right] \leq C\left(\left|x-x^{\prime}\right|^{p}+\left(1+\left[|x| \vee\left|x^{\prime}\right|\right]^{p}\right)\left|t-t^{\prime}\right|\right)$

with some constant $C=C(p, K, T)$.

Proof. They are the standard estimates for the Lipschitz SDEs. See, for example, Theorem 4.1.1 [9]. For the selfcontainedness, we give a proof in Appendix B.3 for regularities.

We are interested in the Markovian anticipated BSDE associated with $\left(X_{v}^{t, x}\right)_{v \in[0, T]}$ :

$$
\begin{aligned}
Y_{s}^{t, x} & =\xi\left(X_{T}^{t, x}\right)+\int_{s}^{T} \mathbf{1}_{r \geq t} \mathbb{E}_{\mathcal{F}_{r}} f\left(r, X_{r}^{t, x},\left(Y_{v}^{t, x}\right)_{v \in[r, T]}, Y_{r}^{t, x}, Z_{r}^{t, x}, \psi_{r}^{t, x}\right) d r \\
& -\int_{s}^{T} Z_{r}^{t, x} d W_{r}-\int_{s}^{T} \int_{E} \psi_{r}^{t, x}(e) \widetilde{\mu}(d r, d e),
\end{aligned}
$$

where $f:[0, T] \times \mathbb{R}^{n} \times \mathbb{D}[0, T] \times \mathbb{R} \times \mathbb{R}^{1 \times d} \times \mathbb{L}^{2}(E, \nu) \rightarrow \mathbb{R}$ and $\xi: \mathbb{R}^{n} \rightarrow \mathbb{R}$ are non-random measurable functions. Note that $\left(Y_{s}^{t, x}, Z_{s}^{t, x}, \psi_{s}^{t, x}\right) \equiv\left(Y_{t}^{t, x}, 0,0\right)$ for $s \leq t$.

Assumption 4.2. (i) The driver $f$ is a map such that for every $(x, y, z, \psi) \in \mathbb{R}^{n} \times \mathbb{R} \times \mathbb{R}^{1 \times d} \times$ $\mathbb{L}^{2}(E, \nu)$ and any càdlàg $\mathbb{F}$-adapted process $\left(Y_{v}\right)_{v \in[0, T]}$, the process $\left(\mathbb{E}_{\mathcal{F}_{t}} f\left(t, x,\left(Y_{v}\right)_{v \in[t, T]}, y, z, \psi\right), t \in\right.$ $[0, T])$ is $\mathbb{F}$-progressively measurable.

(ii) For every $(x, q, y, z, \psi) \in \mathbb{R}^{n} \times \mathbb{D}[0, T] \times \mathbb{R} \times \mathbb{R}^{1 \times d} \times \mathbb{L}^{2}(E, \nu)$, there exist constants $\beta, \delta \geq 0$, $\gamma>0$ and a positive non-random function $l:[0, T] \rightarrow \mathbb{R}$ such that

$$
\begin{aligned}
-l_{t} & -\delta\left(\sup _{v \in[t, T]}\left|q_{v}\right|\right)-\beta|y|-\frac{\gamma}{2}|z|^{2}-\int_{E} j_{\gamma}(-\psi(e)) \nu(d e) \leq f\left(t, x,\left(q_{v}\right)_{v \in[t, T]}, y, z, \psi\right) \\
& \leq l_{t}+\delta\left(\sup _{v \in[t, T]}\left|q_{v}\right|\right)+\beta|y|+\frac{\gamma}{2}|z|^{2}+\int_{E} j_{\gamma}(\psi(e)) \nu(d e)
\end{aligned}
$$

dt-a.e. $t \in[0, T]$, where $j_{\gamma}(u)=\frac{1}{\gamma}\left(e^{\gamma u}-1-\gamma u\right)$.

(iii) $\|\xi(\cdot)\|_{\infty}, \sup _{t \in[0, T]}\left(l_{t}\right)<\infty$.

Assumption 4.3. For each $M>0$, and for every $(x, q, y, z, \psi),\left(x^{\prime}, q^{\prime}, y^{\prime}, z^{\prime}, \psi^{\prime}\right) \in \mathbb{R}^{n} \times$ $\mathbb{D}[0, T] \times \mathbb{R} \times \mathbb{R}^{1 \times d} \times \mathbb{L}^{2}(E, \nu)$ satisfying $|y|,\left|y^{\prime}\right|,\|\psi\|_{\mathbb{L}^{\infty}(\nu)},|| \psi^{\prime} \|_{\mathbb{L}^{\infty}(\nu)}, \sup _{v \in[0, T]}\left|q_{v}\right|, \sup _{v \in[0, T]}\left|q_{v}^{\prime}\right| \leq$ $M$, there exist some positive constants $K_{M}$ (depending on $M$ ) and $K_{\xi} \geq 0, \rho \geq 0, \alpha \in(0,1]$ such that, for $d t$-a.e. $t \in[0, T]$,

$$
\begin{aligned}
\bullet \mid f(t, x, & \left.\left(q_{v}\right)_{v \in[t, T]}, y, z, \psi\right)-f\left(t, x,\left(q_{v}^{\prime}\right)_{v \in[t, T]}, y^{\prime}, z^{\prime}, \psi^{\prime}\right) \mid \\
\leq & K_{M}\left(\sup _{v \in[t, T]}\left|q_{v}-q_{v}^{\prime}\right|+\left|y-y^{\prime}\right|+|| \psi-\psi^{\prime} \|_{\mathbb{L}^{2}(\nu)}\right) \\
& +K_{M}\left(1+|z|+\left|z^{\prime}\right|+|| \psi \|_{\mathbb{L}^{2}(\nu)}+|| \psi^{\prime}||_{\mathbb{L}^{2}(\nu)}\right)\left|z-z^{\prime}\right|, \\
\bullet \mid f(t, x, & \left.\left(q_{v}\right)_{v \in[t, T]}, y, z, \psi\right)-f\left(t, x^{\prime},\left(q_{v}\right)_{v \in[t, T]}, y, z, \psi\right) \mid \\
& \leq K_{M}\left(1+\left[|x| \vee\left|x^{\prime}\right|\right]^{\rho}+|z|^{2}+|| \psi \|_{\mathbb{L}^{2}(\nu)}^{2}\right)\left|x-x^{\prime}\right|^{\alpha},
\end{aligned}
$$

and $\left|\xi(x)-\xi\left(x^{\prime}\right)\right| \leq K_{\xi}\left|x-x^{\prime}\right|^{\alpha}$. 
Proposition 4.1. Under Assumptions 4.1, 4.2 and 4.3, suppose that there exists a bounded solution $\left(Y^{t, x}, Z^{t, x}, \psi^{t, x}\right) \in \mathbb{S}^{\infty} \times \mathbb{H}^{2} \times \mathbb{J}^{2}$ for each $(t, x) \in[0, T] \times \mathbb{R}^{n}$. Then the solution is unique and $\left(Y^{t, x}, Z^{t, x}, \psi^{t, x}\right) \in \mathbb{S}^{\infty} \times \mathbb{H}_{B M O}^{2} \times \mathbb{J}_{B M O}^{2}$ with the norm solely controlled by $A=\left(\|\xi\|_{\infty}\right.$, sup $\left._{t \in[0, T]} l_{t}, \delta, \beta, \gamma, T\right)$, which is, in particular, independent of $(t, x) \in[0, T] \times \mathbb{R}^{n}$.

Moreover, if $Y_{t}^{t, x}$ is a deterministic map in $(t, x)$, the map $u:[0, T] \times \mathbb{R}^{n} \rightarrow \mathbb{R}$ defined by $u(t, x):=Y_{t}^{t, x}$ satisfies for any pair of $(t, x),\left(t^{\prime}, x^{\prime}\right) \in[0, T] \times \mathbb{R}^{n}$,

$$
\left|u(t, x)-u\left(t^{\prime}, x^{\prime}\right)\right| \leq C\left(1+\left[|x| \vee\left|x^{\prime}\right|\right]^{\rho}\right)\left(\left|x-x^{\prime}\right|^{\alpha}+\left(1+\left[|x| \vee\left|x^{\prime}\right|\right]^{\alpha}\right)\left|t-t^{\prime}\right|^{\frac{1}{2 p \bar{q}^{2}}}\right)
$$

with some constant $C=C\left(\alpha, \rho, p, \bar{q}, K_{\xi}, K, K ., A\right)$ for any $p \geq 2$ and $\bar{q} \in\left[q_{*}, \infty\right)$ such that $\alpha p \bar{q}^{2} \geq 1$, where $q_{*}>1$ is some constant determined by $(K ., A)$.

Proof. The first part follows from Lemmas 3.1, 3.2 and Corollary 3.1.

Let us assume $t^{\prime} \leq t$ without loss of any generality. Put $\delta Y:=Y^{t, x}-Y^{t^{\prime}, x^{\prime}}$,

$$
\begin{aligned}
\delta f(r):= & \mathbf{1}_{r \geq t} f\left(r, X_{r}^{t, x},\left(Y_{v}^{t, x}\right)_{v \in[r, T]}, \Theta_{r}^{t, x}\right)-\mathbf{1}_{r \geq t^{\prime}} f\left(r, X_{r}^{t^{\prime}, x^{\prime}},\left(Y_{v}^{t, x}\right)_{v \in[r, T]}, \Theta_{r}^{t, x}\right) \\
= & \mathbf{1}_{r \geq t}\left(f\left(r, X_{r}^{t, x},\left(Y_{v}^{t, x}\right)_{v \in[r, T]}, \Theta_{r}^{t, x}\right)-f\left(r, X_{r}^{t^{\prime}, x^{\prime}},\left(Y_{v}^{t, x}\right)_{v \in[r, T]}, \Theta_{r}^{t, x}\right)\right) \\
& -\mathbf{1}_{t^{\prime} \leq r \leq t} f\left(r, X_{r}^{t^{\prime}, x^{\prime}},\left(Y_{v}^{t, x}\right)_{v \in[r, T]}, Y_{t}^{t, x}, 0,0\right),
\end{aligned}
$$

and $\delta \xi=\xi\left(X_{T}^{t, x}\right)-\xi\left(X_{T}^{t^{\prime}, x^{\prime}}\right)$. By Proposition 3.1, for any $p \geq 2, \bar{q} \in\left[q_{*}, \infty\right)$,

$$
\begin{aligned}
\left|u(t, x)-u\left(t^{\prime}, x^{\prime}\right)\right| & \leq \mathbb{E}\left[\sup _{s \in[0, T]}\left|Y_{s}^{t, x}-Y_{s}^{t^{\prime}, x^{\prime}}\right|^{p}\right]^{\frac{1}{p}} \\
& \leq C \mathbb{E}\left[|\delta \xi|^{p \bar{q}^{2}}+\left(\int_{0}^{T} \mathbb{E}_{\mathcal{F}_{r}}|\delta f(r)| d r\right)^{p \bar{q}^{2}}\right]^{\frac{1}{p \bar{q}^{2}}}
\end{aligned}
$$

with $C=C(p, \bar{q}, K ., A)$. The universal bounds of Lemmas 3.1 and 3.2 imply that $\left\|Y^{t, x}\right\|_{\mathbb{S} \infty}$, $\left\|Z^{t, x}\right\|_{\mathbb{H}_{B M O}^{2}},\left\|\psi^{t, x}\right\|_{\mathbb{J}_{B M O}^{2}} \leq C$ with some $C=C(A)$ uniformly in $(t, x)$. Thus one can apply fixed $K_{M}^{B M}$ for the whole range in Assumption 4.3 provided $M$ is chosen large enough. It follows that

$$
\begin{aligned}
\mathbb{E}_{\mathcal{F}_{r}}|\delta f(r)| & \leq \mathbf{1}_{r \geq t} K_{M}\left(1+\left[\left|X_{r}^{t, x}\right| \vee\left|X_{r}^{t^{\prime}, x^{\prime}}\right|\right]^{\rho}+\left|Z_{r}^{t, x}\right|^{2}+\left\|\psi_{r}^{t, x}\right\|_{\mathbb{L}^{2}(\nu)}^{2}\right)\left|X_{r}^{t, x}-X_{r}^{t^{\prime}, x^{\prime}}\right|^{\alpha} \\
& +\mathbf{1}_{t^{\prime} \leq r \leq t}\left(l+\delta \mathbb{E}_{\mathcal{F}_{r}}\left[|| Y^{t, x} \|_{[r, T]}\right]+\beta\left|Y_{t}^{t, x}\right|\right) .
\end{aligned}
$$

Hence, using the boundedness of $Y^{t, x}$ and Cauchy-Schwartz inequality, one obtains

$$
\begin{aligned}
& \mathbb{E}\left[\left(\int_{0}^{T} \mathbb{E}_{\mathcal{F}_{r}}|\delta f(r)| d r\right)^{p \bar{q}^{2}}\right]^{\frac{1}{p \bar{q}^{2}}} \\
& \leq C \mathbb{E}\left[1+\left[\left\|X^{t, x}\right\|_{[0, T]} \vee\left\|X^{t^{\prime}, x^{\prime}}\right\|_{[0, T]}\right]^{2 \rho p \bar{q}^{2}}+\left(\int_{0}^{T}\left|Z_{r}^{t, x}\right|^{2}+\left\|\psi_{r}^{t, x}\right\|_{\mathbb{L}^{2}(\nu)}^{2} d r\right)^{2 p \bar{q}^{2}}\right]^{\frac{1}{2 p \bar{q}^{2}}} \\
& \quad \times \mathbb{E}\left[\left\|X^{t, x}-X^{t^{\prime}, x^{\prime}}\right\|_{[0, T]}^{2 \alpha p \bar{q}^{2}}\right]^{\frac{1}{2 p \bar{q}^{2}}}+C\left|t-t^{\prime}\right| .
\end{aligned}
$$

Note here that, by the energy inequality 3 , the following relation holds:

$$
\mathbb{E}\left[\left(\int_{0}^{T}\left|Z_{r}^{t, x}\right|^{2}+\left\|\psi_{r}^{t, x}\right\|_{\mathbb{L}^{2}(\nu)}^{2} d r\right)^{2 p \bar{q}^{2}}\right]^{\frac{1}{2 p \bar{q}^{2}}} \leq C
$$

\footnotetext{
${ }^{3}$ See, for example, Lemma 2.2 [16]. As for a simple proof, see Lemma 9.6.5 [8].
} 
where the constant $C$ depends only on $\left(\|Z\|_{\mathbb{H}_{B M O}^{2}},\|\psi\|_{\mathbb{J}_{B M O}^{2}}\right)$ and $p \bar{q}^{2}$. Using Lemma 4.1(a) and (c), one obtains the desired regularity. The contribution from $\delta \xi$ can be computed similarly.

Remark 4.1. Under the conditions of the above proposition, we have, for each $s \in[0, T]$, $Y_{s}^{t, x}=Y_{s}^{s, X_{s}^{t, x}}=u\left(s, X_{s}^{t, x}\right)$ a.s. due to the uniqueness of solution $Y^{t, x}$. Furthermore, since the function $u$ is jointly continuous, $u\left(s, X_{s}^{t, x}\right)_{s \in[0, T]}$ is càdlàg $\mathbb{F}$-adapted. Thus, Chapter 1, Theorem 2 of [33] implies that $Y_{s}^{t, x}=u\left(s, X_{s}^{t, x}\right) \forall s \in[0, T]$ a.s.

We now introduce a sequence of regularized anticipated BSDEs with $m \in \mathbb{N}$ :

$$
\begin{aligned}
Y_{s}^{m, t, x}= & \xi\left(X_{T}^{t, x}\right)+\int_{s}^{T} \mathbf{1}_{r \geq t} \mathbb{E}_{\mathcal{F}_{r}} f_{m}\left(r, X_{r}^{t, x},\left(Y_{v}^{m, t, x}\right)_{v \in[r, T]}, Y_{r}^{m, t, x}, Z_{r}^{m, t, x}, \psi_{r}^{m, t, x}\right) d r \\
& -\int_{s}^{T} Z_{r}^{m, t, x} d W_{r}-\int_{s}^{T} \int_{E} \psi_{r}^{m, t, x}(e) \widetilde{\mu}(d r, d e)
\end{aligned}
$$

where $f_{m}$ is defined by, $\forall(r, x, q, y, z, \psi) \in[0, T] \times \mathbb{R}^{n} \times \mathbb{D}[0, T] \times \mathbb{R} \times \mathbb{R}^{1 \times d} \times \mathbb{L}^{2}(E, \nu)$,

$$
f_{m}\left(r, x,\left(q_{s}\right)_{v \in[r, T]}, y, z, \psi\right):=f\left(r, x,\left(\varphi_{m}\left(q_{s}\right)\right)_{v \in[r, T]}, \varphi_{m}(y), \varphi_{m}(z), \varphi_{m}\left(\psi \circ \zeta_{m}\right)\right) .
$$

Here, we have used a simple truncation function

$$
\varphi_{m}(x):= \begin{cases}-m & \text { for } x \leq-m \\ x & \text { for }|x| \leq m \\ m & \text { for } x \geq m\end{cases}
$$

and a cutoff function $\psi \circ \zeta_{m}(e):=\psi(e) \mathbf{1}_{|e| \geq 1 / m}$, which are applied component-wise for $z, \psi$.

Lemma 4.2. Suppose that the driver $f$ satisfies Assumptions 4.2 and 4.3. Then, $\left(f_{m}\right)_{m \in \mathbb{N}}$ also satisfy Assumptions 4.2 and 4.3 uniformly in $m \in \mathbb{N}$. Moreover, for each $m \in \mathbb{N}$, the driver $f_{m}$ is a.e. bounded and globally Lipschitz continuous with respect to $(q, y, z, \psi)$ in the sense of Assumption C.1.

Proof. With $\left|\varphi_{m}(x)\right| \leq|x|,\left|\varphi_{m}(x)-\varphi_{m}\left(x^{\prime}\right)\right| \leq\left|x-x^{\prime}\right|$ and use the convexity of the function $j_{\gamma}(\cdot)$, the first claim is obvious. By denoting $C_{m}:=\max _{1 \leq k \leq 1} \int_{|e| \geq 1 / m} \nu^{i}(d e)<\infty$, one sees $\left|f_{m}\right| \leq \sup _{t \in[0, T]} l_{t}+(\delta+\beta) m+\frac{\gamma}{2} d m^{2}+k j_{\gamma}(m) C_{m}$ a.e. by the structure condition. By noticing the fact that

$$
\left\|\varphi_{m}\left(\psi \circ \zeta_{m}\right)\right\|_{\mathbb{L}^{2}(\nu)}^{2} \leq \sum_{i=1}^{k} m^{2} \int_{|e| \geq 1 / m} \nu^{i}(d e) \leq k m^{2} C_{m}
$$

the global Lipschitz continuity can be confirmed easily.

Lemma 4.3. There exists a unique solution $\left(Y^{m, t, x}, Z^{m, t, x}, \psi^{m, t, x}\right)$ to 4.4) satisfying

$$
\left\|Y^{m, t, x}\right\|_{\mathbb{S} \infty},\left\|Z^{m, t, x}\right\|_{\mathbb{H}_{B M O}^{2}},\left\|\psi^{m, t, x}\right\|_{\mathbb{J}_{B M O}^{2}} \leq C
$$

with some constant $C=C(A)$, depending only on those relevant for the universal bound, uniformly in $(m, t, x) \in \mathbb{N} \times[0, T] \times \mathbb{R}^{n}$. Moreover, $\left(Y_{s}^{m, t, x}, Z_{s}^{m, t, x}, \psi_{s}^{m, t, x} ; s \in[t, T]\right)$ is adapted to the $\sigma$-algebra $\mathcal{F}_{s}^{t}$ generated by $(W, \mu)$ after $t$, that is, $\mathcal{F}_{s}^{t}=\sigma\left(W_{u}-W_{t}, \mu((t, u], \cdot) ; t \leq u \leq\right.$ $s)$ for each $s \in[t, T]$. In particular, $Y_{t}^{m, t, x}$ is deterministic in $(t, x)$. 
Proof. Thanks to Lemma 4.2, Proposition C.1 is applicable to (4.4), which implies that there exists a unique solution $\left(Y^{m, t, x}, Z^{m, t, x}, \psi^{m, t, x}\right) \in \mathcal{K}^{2}[0, T]$ of (4.4). Since $|\xi|$ and $\left|f_{m}\right|$ are bounded, we actually have $Y^{m, t, x} \in \mathbb{S}^{\infty}$. Therefore, Lemmas 4.2, 3.1 and 3.2 imply the desired bound

$$
\left\|Y^{m, t, x}\right\|_{\mathbb{S} \infty},\left\|Z^{m, t, x}\right\|_{\mathbb{H}_{B M O}^{2}},\left\|\psi^{m, t, x}\right\|_{\mathbb{J}_{B M O}^{2}} \leq C
$$

uniformly in $(m, t, x) \in \mathbb{N} \times[0, T] \times \mathbb{R}^{n}$. This proves the first part.

We can prove the latter claims by following the same idea given in Proposition 4.2 [1] or Theorem 9.5.6 8]. Consider the shifted Brownian motion and Poisson random measure $\left(W^{\prime}, \mu^{\prime}\right)$ defined by $W_{s}^{\prime}:=W_{t+s}-W_{t}, \mu^{\prime}((0, s], \cdot):=\mu((t, t+s], \cdot), 0 \leq s \leq T-t$, as well as their associated filtration $\mathcal{F}_{s}^{\prime}:=\mathcal{F}_{t+s}^{t}$. Let $\left(X_{s}^{\prime(0, x)}, 0 \leq s \leq T-t\right)$ be the solution to the following SDE:

$X_{s}^{\prime(0, x)}=x+\int_{0}^{s} b\left(r+t, X_{r}^{\prime(0, x)}\right) d r+\int_{0}^{s} \sigma\left(r+t, X_{r}^{\prime(0, x)}\right) d W_{r}^{\prime}+\int_{0}^{s} \int_{E} \gamma\left(r+t, X_{r+t-}^{\prime(0, x)}, e\right) \widetilde{\mu}^{\prime}(d r, d e)$

where $\widetilde{\mu}^{\prime}$ is the compensated measure for $\mu^{\prime}$. By the strong uniqueness of the SDE, $X_{s-t}^{\prime(0, x)}=$ $X_{s}^{t, x}$ for $t \leq s \leq T \mathbb{P}$-a.s. Hence $X_{s}^{t, x}$ is $\mathcal{F}_{s}^{t}\left(=\mathcal{F}_{s-t}^{\prime}\right)$-measurable.

Similarly, let us consider the Lipschitz ABSDE for $s \in[0, T-t]$;

$$
\begin{aligned}
Y_{s}^{\prime}= & \xi\left(X_{T-t}^{\prime(0, x)}\right)+\int_{s}^{T-t} \mathbb{E}_{\mathcal{F}_{r}^{\prime}} f_{m}\left(r+t, X_{r}^{\prime(0, x)},\left(Y_{v}^{\prime}\right)_{v \in[r, T-t]}, Y_{r}^{\prime}, Z_{r}^{\prime}, \psi_{r}^{\prime}\right) d r \\
& -\int_{s}^{T-t} Z_{r}^{\prime} d W_{r}^{\prime}-\int_{s}^{T-t} \int_{E} \psi_{r}^{\prime}(e) \widetilde{\mu}^{\prime}(d r, d e)
\end{aligned}
$$

where $\left(Y^{\prime}, Z^{\prime}, \psi^{\prime}\right)$ is the unique solution with respect to the filtration $\left(\mathcal{F}_{s}^{\prime}\right)_{s \in[0, T-t]}$ by Proposition C.1. Note here that the conditional expectation $\mathbb{E}_{\mathcal{F}_{r}^{\prime}}[\cdot]=\mathbb{E}_{\mathcal{F}_{r+t}^{t}}[\cdot]$ applied to the driver can be replaced by $\mathbb{E}_{\mathcal{F}_{r+t}}[\cdot]$ since the arguments of $f_{m}$ are adapted to $\left(\mathcal{F}_{s}^{\prime}\right)_{s \in[0, T-t]}$ and hence independent of $\mathcal{F}_{t}$. Changing the integration variable to $r+t \rightarrow r \in[t, T]$, and using the fact that $d W_{r-t}^{\prime}=d W_{r}$ and $\widetilde{\mu}^{\prime}(d(r-t), d e)=\widetilde{\mu}(d r, d e)$, one obtains

$$
\begin{aligned}
Y_{s}^{\prime}= & \xi\left(X_{T-t}^{\prime(0, x)}\right)+\int_{s+t}^{T} \mathbb{E}_{\mathcal{F}_{r}} f_{m}\left(r, X_{r-t}^{\prime(0, x)},\left(Y_{v-t}^{\prime}\right)_{v \in[r, T]}, Y_{r-t}^{\prime}, Z_{r-t}^{\prime}, \psi_{r-t}^{\prime}\right) d r \\
& -\int_{s+t}^{T} Z_{r-t}^{\prime} d W_{r}-\int_{s+t}^{T} \int_{E} \psi_{r-t}^{\prime}(e) \widetilde{\mu}(d r, d e) .
\end{aligned}
$$

Since $X_{s-t}^{\prime(0, x)}=X_{s}^{t, x}$, one sees that $\left(Y_{s-t}^{\prime}, Z_{s-t}^{\prime}, \psi_{s-t}^{\prime} ; s \in[t, T]\right)$ is a solution to (4.4) on $[t, T]$. Since the Lipschitz ABSDE has a unique solution by Proposition C.1 (Alternatively, one can use the stability result in Proposition [3.1), $\left(Y_{s-t}^{\prime}, Z_{s-t}^{\prime}, \psi_{s-t}^{\prime}\right)=\left(Y_{s}^{m, t, x}, Z_{s}^{m, t, x}, \psi_{s}^{m, t, x}\right)$ a.s. for every $s \in[t, T]$. Thus, $Y_{s}^{m, t, x}$ is $\mathcal{F}_{s}^{t}\left(=\mathcal{F}_{s-t}^{\prime}\right)$-measurable. In particular, $Y_{t}^{m, t, x}$ is $\mathcal{F}_{t}^{t}\left(=\mathcal{F}_{0}^{\prime}\right)$-measurable and hence deterministic by Blumenthal's 0-1 law.

We now provide our first main result.

Theorem 4.1. Under Assumptions 4.1, 4.2 and 4.3, there exists a unique solution $\left(Y^{t, x}, Z^{t, x}, \psi^{t, x}\right) \in$ $\mathbb{S}^{\infty} \times \mathbb{H}_{B M O}^{2} \times \mathbb{J}_{B M O}^{2}$ to the $A B S D E$ (4.2) for each $(t, x) \in[0, T] \times \mathbb{R}^{n}$.

Proof. Since the uniqueness follows from the first part of Proposition 4.1, it suffices to prove the existence. Lemmas 4.2, 4.3 and Proposition 4.1 imply that the deterministic map $u_{m}$ : $[0, T] \times \mathbb{R}^{n} \rightarrow \mathbb{R}$ defined by $u_{m}(t, x):=Y_{t}^{m, t, x}$ satisfies the local Hölder continuity uniformly 
in $m$ with $C=C\left(\alpha, \rho, p, \bar{q}, K_{\xi}, K, K ., A\right)$ such that

$$
\left|u_{m}(t, x)-u_{m}\left(t^{\prime}, x^{\prime}\right)\right| \leq C\left(1+\left[|x| \vee\left|x^{\prime}\right|\right]^{\rho}\right)\left(\left|x-x^{\prime}\right|^{\alpha}+\left(1+\left[|x| \vee\left|x^{\prime}\right|\right]^{\alpha}\right)\left|t-t^{\prime}\right|^{\frac{1}{2 p \bar{q}^{2}}}\right) .
$$

From Lemma 4.3, it is also clear that $\sup _{m \geq 1} \sup _{(t, x) \in[0, T] \times \mathbb{R}^{n}}\left|u_{m}(t, x)\right| \leq C$.

Let us now confirm the compactness result for $\left(u_{m}\right)_{m \in \mathbb{N}}$. By defining the compact set $\mathbb{K}_{j}$ with $j \in \mathbb{N}$ by $\mathbb{K}_{j}:=[0, T] \times \bar{B}_{j}\left(\mathbb{R}^{n}\right) \subset \mathbb{R}^{n+1}$, we have $\bigcup_{j=1}^{\infty} \mathbb{K}_{j}=[0, T] \times \mathbb{R}^{n}$. Here, $\bar{B}_{j}\left(\mathbb{R}^{n}\right)$ is a closed ball in $\mathbb{R}^{n}$ of radius $j$ centered at the origin. Arzelà-Ascoli theorem (see, Section 10.1 [34]) tells that there exists a subsequence $\left(m^{(1)}\right) \subset(m)$ such that, $\exists u^{(1)} \in C\left(\mathbb{K}_{1}\right)$, $\left(u_{m^{(1)}}\right)$ converges uniformly to $u^{(1)}$ on $\mathbb{K}_{1}$. Since the sequence $\left(u_{m^{(1)}}\right)$ is also bounded and equicontinuous, there exists a further subsequence $\left(m^{(2)}\right) \subset\left(m^{(1)}\right)$ such that, $\exists u^{(2)} \in C\left(\mathbb{K}_{2}\right)$, $\left(u_{m^{(2)}}\right)$ converges uniformly to $u^{(2)}$ on $\mathbb{K}_{2}$. By construction, it is clear that $\left.u^{(2)}\right|_{\mathbb{K}_{1}}=u^{(1)}$. Continue the above procedures and construct a diagonal sequence as

$$
\left(m^{(m)}\right)_{m \geq 1}:=\left\{1^{(1)}, 2^{(2)}, \cdots, j^{(j)}, \cdots\right\} .
$$

From Lemma 2 in Section 10.1 [34] implies that there exists a subsequence $\left(m^{\prime}\right) \subset\left(m^{(m)}\right)$ and some function $u:[0, T] \times \mathbb{R}^{n} \rightarrow \mathbb{R}$ such that $\left(u_{m^{\prime}}\right)$ converges to $u$ pointwise on the whole $[0, T] \times \mathbb{R}^{n}$ space. Moreover, the function $u$ is actually continuous i.e. $u \in C\left([0, T] \times \mathbb{R}^{n}\right)$. In fact, by the above construction of the sequence $\left(m^{(m)}\right),\left(u_{m^{\prime}}\right)$ converges uniformly to this function $u$ on any compact subset $\mathbb{K}_{R}$.

In the remainder, we work on the sequence $\left(\mathrm{m}^{\prime}\right)$ (and possibly its further subsequences). Define the càdlàg $\mathbb{F}$-adapted process $\left(Y_{s}^{t, x}\right)_{s \in[0, T]}$ by $Y_{s}^{t, x}:=u\left(s, X_{s}^{t, x}\right), \quad \forall(\omega, s) \in \Omega \times[0, T]$. The uniform boundedness of $\left(u_{m^{\prime}}, u\right)$, Lemma 4.1(a) and Chebyshev's inequality give

$$
\left\|Y^{m^{\prime}, t, x}-Y^{t, x}\right\|_{\mathbb{S}^{p}}^{p} \leq \mathbb{E}\left[\sup _{s \in[0, T]}\left|u_{m^{\prime}}\left(s, X_{s}^{t, x}\right)-u\left(s, X_{s}^{t, x}\right)\right|^{p} \mathbf{1}_{\left\{\sup _{s \in[0, T]}\left|X_{s}^{t, x}\right| \leq R\right\}}\right]+C\left(\frac{1+|x|^{j}}{R^{j}}\right)
$$

for every $R>0$ and $p, j \in \mathbb{N}$ with some $m^{\prime}$-independent constant $C$. For a given $\epsilon>0$, the 2 nd term becomes smaller than $\epsilon / 2$ with $R$ large enough. Since $\left(u_{m^{\prime}}\right)$ converges uniformly to $u$ on any compact set, the first term also becomes smaller than $\epsilon / 2$ for large $m^{\prime}$. Hence, $\left\|Y^{m^{\prime}, t, x}-Y^{t, x}\right\|_{\mathbb{S}^{p}}^{p} \leq \epsilon$ for large $m^{\prime}$. Thus one concludes $Y^{m^{\prime}, t, x} \rightarrow Y^{t, x}$ in $\mathbb{S}^{p}$ for every $p \in \mathbb{N}$. Since it implies $\sup _{s \in[0, T]}\left|Y^{m^{\prime}, t, x}-Y_{s}^{t, x}\right| \rightarrow 0$ as $m^{\prime} \rightarrow \infty$ in probability, extracting further subsequence (still denoted by $\left(m^{\prime}\right)$ ), we have $\lim _{m^{\prime} \rightarrow \infty} \sup _{s \in[0, T]}\left|Y_{s}^{m^{\prime}, t, x}-Y_{s}^{t, x}\right|=0 \mathbb{P}$-a.s. In particular, it means $\left\|Y^{m^{\prime}, t, x}-Y^{t, x}\right\|_{\mathbb{S}^{\infty}} \rightarrow 0$. It also implies that $\left(Y^{m^{\prime}, t, x}\right)_{m^{\prime}}$ forms a Cauchy sequence in $\mathbb{S}^{\infty}$.

With $m_{1}, m_{2} \in\left(m^{\prime}\right)$, let us put $\delta Y^{m_{1}, m_{2}}:=Y^{m_{1}, t, x}-Y^{m_{2}, t, x}, \delta Z^{m_{1}, m_{2}}:=Z^{m_{1}, t, x}-Z^{m_{2}, t, x}$ and $\delta \psi^{m_{1}, m_{2}}:=\psi^{m_{1}, t, x}-\psi^{m_{2}, t, x}$. Ito formula applied to $\left|\delta Y_{t}^{m_{1}, m_{2}}\right|^{2}$ yields for any $\tau \in \mathcal{T}_{0}^{T}$,

$$
\begin{gathered}
\mathbb{E}_{\mathcal{F}_{\tau}}\left[\left|\delta Y_{\tau}^{m_{1}, m_{2}}\right|^{2}+\int_{\tau}^{T}\left|\delta Z_{r}^{m_{1}, m_{2}}\right|^{2} d r+\int_{\tau}^{T} \int_{E}\left|\delta \psi_{r}^{m_{1}, m_{2}}(e)\right|^{2} \mu(d r, d e)\right] \\
=\mathbb{E}_{\mathcal{F}_{\tau}}\left[\int _ { \tau \vee t } ^ { T } 2 \delta Y _ { r } ^ { m _ { 1 } , m _ { 2 } } \mathbb { E } _ { \mathcal { F } _ { r } } \left[f_{m_{1}}\left(r, X_{r}^{t, x},\left(Y_{v}^{m_{1}, t, x}\right)_{v \in[r, T]}, \Theta_{r}^{m_{1}, t, x}\right)\right.\right. \\
\left.\left.\quad-f_{m_{2}}\left(r, X_{r}^{t, x},\left(Y_{v}^{m_{2}, t, x}\right)_{v \in[r, T]}, \Theta_{r}^{m_{2}, t, x}\right)\right] d r\right]
\end{gathered}
$$


and hence

$$
\begin{aligned}
& \mathbb{E}_{\mathcal{F}_{\tau}} \int_{\tau}^{T}\left|\delta Z_{r}^{m_{1}, m_{2}}\right|^{2} d r+\mathbb{E}_{\mathcal{F}_{\tau}} \int_{\tau}^{T} \|\left.\delta \psi_{r}^{m_{1}, m_{2}}\right|_{\mathbb{L}^{2}(\nu)} ^{2} d r \\
& \leq 2|| \delta Y^{m_{1}, m_{2}} \|_{\mathbb{S} \infty} \mathbb{E}_{\mathcal{F}_{\tau}} \int_{\tau}^{T} \sum_{i=1}^{2}\left|f_{m}\left(r, X_{r}^{t, x},\left(Y_{v}^{m_{i}, t, x}\right)_{v \in[r, T]}, \Theta_{r}^{m_{i}, t, x}\right)\right| d r .
\end{aligned}
$$

From Lemma 4.2 and Assumption 4.3, the conditional expectation of the 2nd line is bounded by $C \sum_{i=1}^{2}\left(1+\left\|Y^{m_{i}, t, x}\right\|_{\mathbb{S}^{\infty}}+\left\|Z^{m_{i}, t, x}\right\|_{\mathbb{H}_{B M O}^{2}}^{2}+\left\|\psi^{m_{i}, t, x}\right\|_{\mathbb{J}_{B M O}^{2}}^{2}\right) \leq C$, with $C=C(K ., A)$. Thus the right-hand side converges to zero as $m_{1}, m_{2} \rightarrow \infty$ uniformly in $\tau \in \mathcal{T}_{0}^{T}$. Therefore $\exists\left(Z^{t, x}, \psi^{t, x}\right) \in \mathbb{H}_{B M O}^{2} \times \mathbb{J}_{B M O}^{2}$ such that $Z^{m^{\prime}, t, x} \rightarrow Z^{t, x}$ in $\mathbb{H}_{B M O}^{2}$ and $\psi^{m^{\prime}, t, x} \rightarrow \psi^{t, x}$ in $\mathbb{J}_{B M O}^{2}$.

Proving that $\left(Y^{t, x}, Z^{t, x}, \psi^{t, x}\right)$ provides a solution of (4.2) can be done via the common strategy for the BSDEs. The above convergence results imply, a fortiori, that $Z^{m^{\prime}, t, x} \rightarrow Z^{t, x}$ in $\mathbb{H}^{2}$ and $\psi^{m^{\prime}, t, x} \rightarrow \psi^{t, x}$ in $\mathbb{J}^{2}$. Thus we also have the convergence in measure for $Z^{m^{\prime}, t, x} \rightarrow Z^{t, x}$ and $\psi^{m^{\prime}, t, x} \rightarrow \psi^{t, x}$ with respect to $d \mathbb{P} \otimes d t$ and $d \mathbb{P} \otimes \nu(d e) \otimes d t$, respectively. As we have seen before, we also have $\sup _{s \in[0, T]}\left|Y^{m^{\prime}, t, x}-Y_{s}^{t, x}\right| \rightarrow 0$ in probability. By, for example, Corollary 6.13 [22] (treating general measure space with a $\sigma$-finite measure), there exists a subsequence (still denoted by $\left(m^{\prime}\right)$ ) that yields almost everywhere convergence for the associated measure. Therefore, one has $\sup _{s \in[0, T]}\left|Y_{s}^{m^{\prime}, t, x}-Y_{s}^{t, x}\right| \rightarrow 0$ a.s., $Z^{m^{\prime}, t, x} \rightarrow Z^{t, x} d \mathbb{P} \otimes d s$-a.e. and $\psi^{m, t, x} \rightarrow \psi^{t, x} d \mathbb{P} \otimes \nu(d e) \otimes d s$-a.e.

Since $f_{m} \rightarrow f$ locally uniformly, the above a.e. convergences and the Lipschitz continuity of the driver yields

$$
f_{m^{\prime}}\left(s, X_{s}^{t, x},\left(Y_{v}^{m^{\prime}, t, x}\right)_{v \in[s, T]}, \Theta_{s}^{m^{\prime}, t, x}\right) \rightarrow f\left(s, X_{s}^{t, x},\left(Y_{v}^{t, x}\right)_{v \in[s, T]}, \Theta_{s}^{t, x}\right)
$$

$d \mathbb{P} \otimes d s$-a.e. In order to use the Lebesgue's dominated convergence theorem, we first show that there exists an appropriate subsequence $\left(m^{\prime}\right)$ such that $G:=\sup _{m^{\prime}}\left|Z^{m^{\prime}, t, x}\right|^{2}$ and $H:=$ $\sup _{m^{\prime}}\left\|\psi^{m^{\prime}, t, x}\right\|_{\mathbb{L}^{2}(\nu)}^{2}$ are in $\mathbb{L}^{1}(\Omega \times[0, T])$. Let us follow the idea of Lemma 2.5 in [23]. Since $\left(Z^{m^{\prime}, t, x}\right)$ is a Cauchy sequence in $\mathbb{H}^{2}$, one can extract a subsequence $\left(m_{k}^{\prime}\right)_{k \in \mathbb{N}}$ such that for any $k \in \mathbb{N},\left\|Z^{m_{k+1}^{\prime}, t, x}-Z^{m_{k}^{\prime}, t, x}\right\|_{\mathbb{H}^{2}} \leq 2^{-k}$. On the other hand, for any $s \in[0, T]$, one easily sees that

$$
\sup _{k \in \mathbb{N}}\left|Z_{s}^{m_{k}^{\prime}, t, x}\right| \leq\left|Z_{s}^{m_{1}^{\prime}, t, x}\right|+\sum_{k \in \mathbb{N}}\left|Z_{s}^{m_{k+1}^{\prime}, t, x}-Z_{s}^{m_{k}^{\prime}, t, x}\right| .
$$

Taking the $\mathbb{H}^{2}$-norm in the both side and using Minkowski's inequality,

$$
\begin{aligned}
\mathbb{E}\left[\int_{0}^{T} \sup _{k \in \mathbb{N}}\left|Z_{s}^{m_{k}^{\prime}, t, x}\right|^{2} d s\right]^{\frac{1}{2}} & \leq\left\|Z^{m_{1}^{\prime}, t, x}\right\|_{\mathbb{H}^{2}}+\sum_{k \in \mathbb{N}}\left\|Z^{m_{k+1}^{\prime}, t, x}-Z^{m_{k}^{\prime}, t, x}\right\|_{\mathbb{H}^{2}} \\
& \leq\left\|Z^{m_{1}^{\prime}, t, x}\right\|_{\mathbb{H}^{2}}+1<\infty .
\end{aligned}
$$

Relabeling the subsequence by $\left(m^{\prime}\right)$, one obtains the desired result for $G$. Exactly the same method proves the integrability also for $H$. Now, since $\left|f_{m^{\prime}}\right| \leq C(1+G+H)$ a.s.with some $C=C(K, A)$, we have

$$
\int_{0}^{T}\left|f_{m^{\prime}}\left(r, X_{r}^{t, x},\left(Y_{v}^{m^{\prime}, t, x}\right)_{v \in[r, T]}, \Theta_{r}^{m^{\prime}, t, x}\right)-f\left(r, X_{r}^{t, x},\left(Y_{v}^{t, x}\right)_{v \in[r, T]}, \Theta_{r}^{t, x}\right)\right| d r \rightarrow 0 \text { a.s. }
$$

by the Lebesgue's dominated convergence theorem.

Finally, the BDG inequality and the same arguments using the convergence in probability measure also give $\sup _{s \in[0, T]}\left|\int_{s}^{T}\left(Z_{r}^{m^{\prime}, t, x}-Z_{r}^{t, x}\right) d W_{r}\right| \rightarrow 0$ a.s. and $\sup _{s \in[0, T]} \mid \int_{s}^{T} \int_{E}\left(\psi_{r}^{m^{\prime}, t, x}(e)-\right.$ 
$\left.\psi_{r}^{t, x}(e)\right) \widetilde{\mu}(d r, d e) \mid \rightarrow 0$ a.s. under appropriate subsequences, which guarantees the convergence for the stochastic integration. This finishes the proof.

Remark 4.2. By Theorem 4.1 as well as the uniqueness of the solution, $Y_{t}^{t, x}$ is in fact deterministic in $(t, x)$.

Remark 4.3. In the above proof of Theorem 4.1, the convergence actually occurs in the entire sequence of $(m)$ not only the subsequence $\left(m^{\prime}\right)$. If this is not the case, there must be a subsequence $(\hat{m}) \subset(m)$ such that $\left\|Y^{m_{j}, t, x}-Y^{t, x}\right\|_{\mathbb{S}_{\infty}}>c$ with some $c>0$ for every $m_{j} \in(\hat{m})$. However, by repeating the same procedures done in the proof, we can extract a further subsequence $\left(\hat{m}^{\prime}\right) \subset(\hat{m})$ such that, $\exists\left(\widetilde{Y}^{t, x}, \widetilde{Z}^{t, x}, \widetilde{\psi}^{t, x}\right),\left(Y^{m_{j}, t, x}, Z^{m_{j}, t, x}, \psi^{m_{j}, t, x}\right) \rightarrow$ $\left(\widetilde{Y}^{t, x}, \widetilde{Z}^{t, x}, \widetilde{\psi}^{t, x}\right)$ in $\mathbb{S}^{\infty} \times \mathbb{H}_{B M O}^{2} \times \mathbb{J}_{B M O}^{2}$ as $\left(\hat{m}^{\prime}\right) \ni m_{j} \rightarrow \infty$. One can show that it also provides the solution to (4.2). By the uniqueness of solution, $\tilde{Y}^{t, x}=Y^{t, x}$ in $\mathbb{S}^{\infty}$, which contradicts the assumption.

\section{Some regularity results}

Due to the general path-dependence of $\left(Y_{v}\right)_{v \leq T}$ in the driver, it is difficult to establish Malliavin's differentiability. Interestingly, we can apply the method similar to Lemma 15 in Fromm \& Imkeller (2013) [14] or Lemma 2.5.14 in Fromm (2014) [15] to derive some useful regularity results on the control variables. The method only needs the fundamental Lebesgue's differentiation theorem. 4

Lemma 5.1. Under Assumptions 4.1, 4.2 and 4.3 with $\alpha=1$, the control variables of the solution to the ABSDE (4.2) satisfy the estimate for every $(t, x)$

$$
\left|Z_{s}^{t, x}\right| \leq C\left(1+\left|X_{s}^{t, x}\right|^{1+\rho}\right), \quad \|\left.\psi_{s}^{t, x}\right|_{\mathbb{L}^{2}(\nu)} \leq C\left(1+\left|X_{s-}^{t, x}\right|^{1+\rho}\right)
$$

for $d \mathbb{P} \otimes$ ds-a.e. $(\omega, s) \in \Omega \times[0, T]$ with some constant $C=C\left(\rho, K_{\xi}, K, K ., A\right)$.

Proof. For notational simplicity, let us fix the initial data $(t, x)$ and omit the associated superscripts in the remainder of the proof. We start from the regularized ABSDE (4.4). Choose any $s^{\prime} \in[0, T)$ and define $\delta W_{s}:=W_{s}-W_{s^{\prime}}$ for $s \in\left[s^{\prime}, T\right]$. An application of Ito formula to $\left(Y^{m} \delta W^{\top}\right)$ yields

$$
\begin{aligned}
Y_{s}^{m} \delta W_{s}^{\top} & =\int_{s^{\prime}}^{s} Z_{r}^{m} d r-\int_{s^{\prime}}^{s} \mathbf{1}_{r \geq t} \delta W_{r}^{\top} \mathbb{E}_{\mathcal{F}_{r}} f_{m}\left(r, X_{r},\left(Y_{v}^{m}\right)_{v \in[r, T]}, \Theta_{r}^{m}\right) d r \\
& +\int_{s^{\prime}}^{s} \delta W_{r}^{\top} Z_{r}^{m} d W_{r}+\int_{s^{\prime}}^{s} \int_{E} \delta W_{r}^{\top} \psi_{r}^{m}(e) \widetilde{\mu}(d r, d e)+\int_{s^{\prime}}^{s} Y_{r}^{m} d W_{r}^{\top} .
\end{aligned}
$$

Since $\left(Y^{m}, Z^{m}, \psi^{m}\right) \in \mathbb{S}^{\infty} \times \mathbb{H}_{B M O}^{2} \times \mathbb{J}_{B M O}^{2}$, one can show easily that the last three terms are true martingales. Notice that

$$
\begin{aligned}
& \mathbb{E}\left[\int_{0}^{T}\left|\mathbf{1}_{r \geq t} W_{r}^{\top} \mathbb{E}_{\mathcal{F}_{r}} f_{m}\left(r, X_{r},\left(Y_{v}^{m}\right)_{v \in[r, T]}, \Theta_{r}^{m}\right)\right| d r\right] \\
& \leq C \mathbb{E}\left[\|W\|_{[0, T]}^{2}\right]^{\frac{1}{2}} \mathbb{E}\left[\left(\int_{0}^{T}\left(1+\left|Z_{r}^{m}\right|^{2}+\left\|\psi_{r}^{m}\right\|_{\mathbb{L}^{2}(\nu)}^{2}\right) d r\right)^{2}\right]^{\frac{1}{2}} \leq C
\end{aligned}
$$

\footnotetext{
${ }^{4}$ See, for example, Section E.4, Theorem 6 [13.
} 
with $C=C(K ., A)$. Thus Lebesgue's differentiation theorem implies that,

$$
\begin{gathered}
\lim _{s \downarrow s^{\prime}} \frac{1}{s-s^{\prime}} \int_{s^{\prime}}^{s} \mathbf{1}_{r \geq t} W_{r}^{\top} \mathbb{E}_{\mathcal{F}_{r}} f_{m}\left(r, X_{r},\left(Y_{v}^{m}\right)_{v \in[r, T]}, \Theta_{r}^{m}\right) d r \\
\quad=\mathbf{1}_{s^{\prime} \geq t} W_{s^{\prime}}^{\top} \mathbb{E}_{\mathcal{F}_{s^{\prime}}} f_{m}\left(s^{\prime}, X_{s^{\prime}},\left(Y_{v}^{m}\right)_{v \in\left[s^{\prime}, T\right]}, \Theta_{s^{\prime}}^{m}\right) \text { a.s. }
\end{gathered}
$$

for $d t$-a.e. $s^{\prime} \in[0, T)$. Similarly one obtains for $d t$-a.e. $s^{\prime} \in[0, T)$,

$$
\begin{aligned}
& \lim _{s \downarrow s^{\prime}} \frac{1}{s-s^{\prime}} \int_{s^{\prime}}^{s} Z_{r}^{m} d r=Z_{s^{\prime}}^{m} \text { a.s. } \\
& \lim _{s \downarrow s^{\prime}} \frac{1}{s-s^{\prime}} \int_{s^{\prime}}^{s} \mathbf{1}_{r \geq t} \mathbb{E}_{\mathcal{F}_{r}} f_{m}\left(r, X_{r},\left(Y_{v}^{m}\right)_{v \in[r, T]}, \Theta_{r}^{m}\right) d r \\
& \quad=\mathbf{1}_{s^{\prime} \geq t} \mathbb{E}_{\mathcal{F}_{s^{\prime}}} f_{m}\left(s^{\prime}, X_{s^{\prime}},\left(Y_{v}^{m}\right)_{v \in\left[s^{\prime}, T\right]}, \Theta_{s^{\prime}}^{m}\right) d r \text { a.s. }
\end{aligned}
$$

Since $Z^{m} \in \mathbb{H}^{2}$, we can also take $s^{\prime}$ such that $\mathbb{E}\left[\left|Z_{s^{\prime}}^{m}\right|\right]<\infty$ a.e. in $[0, T)$.

As in Lemma 2.5.14 of [15], we introduce the stopping time $\tau: \Omega \rightarrow\left(s^{\prime}, T\right]$ such that the following inequalities hold for all $s \in\left(s^{\prime}, T\right]$ :

$$
\begin{aligned}
& \text { - }\left|\frac{1}{s-s^{\prime}} \int_{s^{\prime}}^{\tau \wedge s} Z_{r}^{m} d r\right| \leq\left|Z_{s^{\prime}}^{m}\right|+1 \quad \text { a.s. } \\
& \text { - }\left|\frac{1}{s-s^{\prime}} \int_{s^{\prime}}^{\tau \wedge s} \mathbf{1}_{r \geq t} \mathbb{E}_{\mathcal{F}_{r}} f_{m}\left(r, X_{r},\left(Y_{v}^{m}\right)_{v \in[r, T]}, \Theta_{r}^{m}\right) d r\right| \\
& \quad \leq \mathbf{1}_{s^{\prime} \geq t}\left|\mathbb{E}_{\mathcal{F}_{s^{\prime}}} f_{m}\left(s^{\prime}, X_{s^{\prime}},\left(Y_{v}^{m}\right)_{v \in\left[s^{\prime}, T\right]}, \Theta_{s^{\prime}}^{m}\right)\right|+1 \quad \text { a.s. } \\
& \text { - }\left|\frac{1}{s-s^{\prime}} \int_{s^{\prime}}^{\tau \wedge s} \mathbf{1}_{r \geq t} W_{r}^{\top} \mathbb{E}_{\mathcal{F}_{r}} f_{m}\left(r, X_{r},\left(Y_{v}^{m}\right)_{v \in[r, T]}, \Theta_{r}^{m}\right) d r\right| \\
& \quad \leq \mathbf{1}_{s^{\prime} \geq t}\left|W_{s^{\prime}}^{\top} \mathbb{E}_{\mathcal{F}_{s^{\prime}}} f_{m}\left(s^{\prime}, X_{s^{\prime}},\left(Y_{v}^{m}\right)_{v \in\left[s^{\prime}, T\right]}, \Theta_{s^{\prime}}^{m}\right)\right|+1 \quad \text { a.s. }
\end{aligned}
$$

Then one can show from (5.1) and the fact that $\tau(\omega) \wedge s=s$ for sufficiently small $s \in\left(s^{\prime}, T\right]$,

$$
Z_{s^{\prime}}^{m}=\lim _{s \downarrow s^{\prime}} \mathbb{E}_{\mathcal{F}_{s^{\prime}}}\left[\frac{1}{s-s^{\prime}} Y_{\tau \wedge s}^{m}\left(W_{\tau \wedge s}-W_{s^{\prime}}\right)^{\top}\right]
$$

$d \mathbb{P} \otimes d t$-a.e. $\left(\omega, s^{\prime}\right) \in \Omega \times[0, T)$ by the dominated convergence theorem. One sees

$$
\begin{aligned}
& \left|\mathbb{E}_{\mathcal{F}_{s^{\prime}}}\left[\frac{1}{s-s^{\prime}} Y_{\tau \wedge s}^{m}\left(W_{\tau \wedge s}-W_{s^{\prime}}\right)^{\top}\right]\right| \\
& \leq\left|\mathbb{E}_{\mathcal{F}_{s^{\prime}}}\left[\frac{1}{s-s^{\prime}} Y_{s}^{m}\left(W_{\tau \wedge s}-W_{s^{\prime}}\right)^{\top}\right]\right|+\left|\mathbb{E}_{\mathcal{F}_{s^{\prime}}}\left[\frac{1}{s-s^{\prime}}\left(Y_{s}^{m}-Y_{\tau \wedge s}^{m}\right)\left(W_{\tau \wedge s}-W_{s^{\prime}}\right)^{\top}\right]\right|,
\end{aligned}
$$

where the second term yields

$$
\begin{aligned}
& \left|\mathbb{E}_{\mathcal{F}_{s^{\prime}}}\left[\frac{1}{s-s^{\prime}}\left(Y_{s}^{m}-Y_{\tau \wedge s}^{m}\right)\left(W_{\tau \wedge s}-W_{s^{\prime}}\right)^{\top}\right]\right| \\
& =\mathbb{E}_{\mathcal{F}_{s^{\prime}}}\left[\frac{1}{s-s^{\prime}} \mathbb{E}_{\mathcal{F}_{\tau \wedge s}}\left[Y_{s}^{m}-Y_{\tau \wedge s}^{m}\right]\left(W_{\tau \wedge s}-W_{s^{\prime}}\right)^{\top}\right] \mid \\
& \leq \mathbb{E}_{\mathcal{F}_{s^{\prime}}}\left[\frac{1}{s-s^{\prime}} \int_{\tau \wedge s}^{s} \mathbb{E}_{\mathcal{F}_{\tau \wedge s}}\left|f_{m}\left(r, X_{r},\left(Y_{v}^{m}\right)_{v \in[r, T]}, \Theta_{r}^{m}\right)\right| d r\left(W_{\tau \wedge s}-W_{s^{\prime}}\right)^{\top}\right] \\
& \leq C_{m} \mathbb{E}_{\mathcal{F}_{s^{\prime}}}\left[\left|W_{\tau \wedge s}-W_{s^{\prime}}\right|^{2}\right]^{\frac{1}{2}} \leq C_{m} \sqrt{s-s^{\prime}} \rightarrow 0 \quad s \downarrow s^{\prime} .
\end{aligned}
$$


Here, we have used the fact that $\left|f_{m}\right|$ is essentially bounded for each $m$ (see Lemma 4.2). The first term gives the estimate with some constant $C$ independent of $m$ such that

$$
\begin{aligned}
& \left|\mathbb{E}_{\mathcal{F}_{s^{\prime}}}\left[\frac{1}{s-s^{\prime}} u_{m}\left(s, X_{s}\right)\left(W_{\tau \wedge s}-W_{s^{\prime}}\right)^{\top}\right]\right|=\left|\mathbb{E}_{\mathcal{F}_{s^{\prime}}}\left[\frac{1}{s-s^{\prime}}\left(u_{m}\left(s, X_{s}\right)-u_{m}\left(s, X_{s^{\prime}}\right)\right)\left(W_{\tau \wedge s}-W_{s^{\prime}}\right)^{\top}\right]\right| \\
& \leq \frac{1}{\sqrt{s-s^{\prime}}} \mathbb{E}_{\mathcal{F}_{s^{\prime}}}\left[\left|u_{m}\left(s, X_{s}\right)-u_{m}\left(s, X_{s^{\prime}}\right)\right|^{2}\right]^{\frac{1}{2}} \quad(\text { by Cauchy-Schwartz) } \\
& \left.\leq \frac{C}{\sqrt{s-s^{\prime}}} \mathbb{E}_{\mathcal{F}_{s^{\prime}}}\left[\left(1+\left[\left|X_{s}\right| \vee\left|X_{s^{\prime}}\right|\right]^{2 \rho}\right)\left|X_{s}-X_{s^{\prime}}\right|^{2}\right]^{\frac{1}{2}} \quad \text { (by (4.6) with } \alpha=1\right) \\
& \leq \frac{C}{\sqrt{s-s^{\prime}}} \mathbb{E}_{\mathcal{F}_{s^{\prime}}}\left[\left(1+\left|X_{s^{\prime}}\right|^{2 \rho}+\left|X_{s}-X_{s^{\prime}}\right|^{2 \rho}\right)\left|X_{s}-X_{s^{\prime}}\right|^{2}\right]^{\frac{1}{2}} \quad(\text { by }|x| \vee|y| \leq|x-y|+|y|) \\
& \leq \frac{C}{\sqrt{s-s^{\prime}}}\left\{\left(1+\left|X_{s^{\prime}}\right|^{\rho}\right) \mathbb{E}_{\mathcal{F}_{s^{\prime}}}\left[\left|X_{s}-X_{s^{\prime}}\right|^{2}\right]^{\frac{1}{2}}+\mathbb{E}_{\mathcal{F}_{s^{\prime}}}\left[\left|X_{s}-X_{s^{\prime}}\right|^{2(1+\rho)}\right]^{\frac{1}{2}}\right\} \\
& \leq C\left(1+\left|X_{s^{\prime}}\right|^{1+\rho}\right) \quad \text { a.s. }
\end{aligned}
$$

where, in the last inequality, we have used a conditional version of Lemma 4.1(b) with the initial value $X_{s^{\prime}}$. Thus we have $d \mathbb{P} \otimes d t$-a.e.

$$
\left|Z_{s^{\prime}}^{m}\right| \leq C\left(1+\left|X_{s^{\prime}}\right|^{1+\rho}\right)
$$

with $C=C\left(\rho, K_{\xi}, K, K ., A\right)$ uniformly in $m$. It is known from the proof of Theorem 4.1 that $Z^{m} \rightarrow Z d \mathbb{P} \otimes d t$-a.e. under an appropriate subsequence, and hence the first claim follows.

The joint continuity of $u$ implies $Y_{s-}=\lim _{r \uparrow s} u\left(r, X_{r}\right)=u\left(s, X_{s-}\right)$ and hence

$$
\begin{aligned}
\int_{E}\left|\psi_{s}(e)\right|^{2} \nu(d e) & =\int_{E}\left|u\left(s, X_{s-}+\gamma\left(s, X_{s-}, e\right)\right)-u\left(s, X_{s-}\right)\right|^{2} \nu(d e) \\
& \leq C \int_{E}\left(1+\left|X_{s-}\right|^{2 \rho}+\left|\gamma\left(s, X_{s-}, e\right)\right|^{2 \rho}\right)\left|\gamma\left(s, X_{s-}, e\right)\right|^{2} \nu(d e) \\
& \leq C\left(1+\left|X_{s-}\right|^{2(1+\rho)}\right) \int_{E}|e|^{2} \nu(d e) \leq C\left(1+\left|X_{s-}\right|^{2(1+\rho)}\right),
\end{aligned}
$$

which proves the second claim.

\section{A non-Markovian setting}

\subsection{Existence}

In order to obtain the existence result in a non-Markovian setting, we need an additional so-called $A_{\Gamma}$-condition on the driver, which is rather restrictive but plays a crucial role in almost every existing work on quadratic growth BSDEs with jumps.

Assumption 6.1. For each $M>0$, for every $q \in \mathbb{D}[0, T], y \in \mathbb{R}, z \in \mathbb{R}^{1 \times d}, \psi, \psi^{\prime} \in \mathbb{L}^{2}(E, \nu)$ with $\sup _{v \in[0, T]}\left|q_{v}\right|,|y|,\|\psi\|_{\mathbb{L}^{\infty}(\nu)},\left\|\psi^{\prime}\right\|_{\mathbb{L}^{\infty}(\nu)} \leq M$ there exists a $\mathcal{P} \otimes \mathcal{E}$-measurable process $\Gamma^{q, y, z, \psi, \psi^{\prime}, M}$ such that, $d \mathbb{P} \otimes d t$-a.e.,

$$
f\left(t,\left(q_{v}\right)_{v \in[t, T]}, y, z, \psi\right)-f\left(t,\left(q_{v}\right)_{v \in[t, T]}, y, z, \psi^{\prime}\right) \leq \int_{E} \Gamma_{t}^{q, y, z, \psi, \psi^{\prime}, M}(e)\left(\psi(e)-\psi^{\prime}(e)\right) \nu(d e)
$$

with $C_{M}^{1}(1 \wedge|e|) \leq \Gamma_{t}^{q, y, z, \psi, \psi^{\prime}, M}(e) \leq C_{M}^{2}(1 \wedge|e|)$ with two $M$ dependent constants satisfying $C_{M}^{1}>-1$ and $C_{M}^{2} \geq 0$. 
We introduce a regularized ABSDE with some positive constant $m>0$ :

$$
\begin{aligned}
Y_{t}^{m}= & \xi+\int_{t}^{T} \mathbb{E}_{\mathcal{F}_{r}} f_{m}\left(r,\left(Y_{v}^{m}\right)_{v \in[r, T]}, Y_{r}^{m}, Z_{r}^{m}, \psi_{r}^{m}\right) d r \\
& -\int_{t}^{T} Z_{r}^{m} d W_{r}-\int_{t}^{T} \int_{E} \psi_{r}^{m}(e) \widetilde{\mu}(d r, d e), t \in[0, T]
\end{aligned}
$$

with the definition $f_{m}\left(t,\left(q_{v}\right)_{v \in[t, T]}, y, z, \psi\right):=f\left(t,\left(\varphi_{m}\left(q_{v}\right)\right)_{v \in[t, T]}, y, z, \psi\right)$ for every $(\omega, t, q, y, z, \psi) \in$ $\Omega \times[0, T] \times \mathbb{D}[0, T] \times \mathbb{R} \times \mathbb{R}^{1 \times d} \times \mathbb{L}^{2}(E, \nu) . \varphi_{m}$ is the truncation function used previously.

Lemma 6.1. If the driver $f$ satisfies Assumptions [3.1, 3.2 and 6.1, then the driver $f_{m}$ defined above also satisfies the same conditions uniformly in $m$. Moreover, if there exists a bounded solution $\left(Y^{m}, Z^{m}, \psi^{m}\right) \in \mathbb{S}^{\infty} \times \mathbb{H}^{2} \times \mathbb{J}^{2}$ to the ABSDE (6.1), then it is unique and belongs to $\mathbb{S}^{\infty} \times \mathbb{H}_{B M O}^{2} \times \mathbb{J}_{B M O}^{2}$ with the norms $\left\|Y^{m}\right\|_{\mathbb{S}^{\infty},},\left\|Z^{m}\right\|_{\mathbb{H}_{B M O}^{2}},\left\|\psi^{m}\right\|_{\mathbb{J}_{B M O}^{2}} \leq C$ with some constant $C$ depending only on $A=\left(\|\xi\|_{\infty},\|l\|_{\mathbb{S} \infty}, \delta, \beta, \gamma, T\right)$.

Proof. The first claim is obvious. The second claim follows from Lemmas $\underline{3.1}, 3.2$ and Corollary 3.1 .

Theorem 6.1. Under Assumptions 3.1, 3.2 and 6.1, there exists a unique solution $(Y, Z, \psi) \in$ $\mathbb{S}^{\infty} \times \mathbb{H}_{B M O}^{2} \times \mathbb{J}_{B M O}^{2}$ to the $A B S D E$ (3.1).

Proof. Uniqueness follows from Corollary 3.1. Notice that it suffices to prove the existence of solution $\left(Y^{m}, Z^{m}, \psi^{m}\right) \in \mathbb{S}^{\infty} \times \mathbb{H}_{B M O}^{2} \times \mathbb{J}_{B M O}^{2}$ of (6.1) for each $m$. In fact, by choosing $m$ bigger than the bound given in Lemma 3.2, one sees $\left(Y^{m}, Z^{m}, \psi^{m}\right)$ actually provides the solution for (3.1). Let fix such an $m$ in the remainder.

Let us put $Y^{m, 0} \equiv 0$ and define a sequence of BSDEs with $n \in \mathbb{N}$ such that

$$
\begin{gathered}
Y_{t}^{m, n}=\xi+\int_{t}^{T} \mathbb{E}_{\mathcal{F}_{r}} f_{m}\left(r,\left(Y_{v}^{m, n-1}\right)_{v \in[r, T]}, Y_{r}^{m, n}, Z_{r}^{m, n}, \psi_{r}^{m, n}\right) d r \\
-\int_{t}^{T} Z_{r}^{m, n} d W_{r}-\int_{t}^{T} \int_{E} \psi_{r}^{m, n}(e) \widetilde{\mu}(d r, d e), t \in[0, T] .
\end{gathered}
$$

The driver for the BSDE (6.2) can be seen as $\widetilde{f}_{m}(r, y, z, \psi):=\mathbb{E}_{\mathcal{F}_{r}} f\left(r,\left(Y_{v}^{m, n-1}\right)_{v \in[r, T]}, y, z, \psi\right)$. By replacing $l_{r}$ by $l_{r}+\delta m$, one sees the data $\left(\xi, \widetilde{f}_{m}\right)$ satisfy Assumptions $3.1,3.2$ and 4.1 in [16] for non-anticipated quadratic-exponential growth BSDEs. Therefore, Theorem 4.1 [16] implies that there exists a (unique) solution $\left(Y^{m, n}, Z^{m, n}, \psi^{m, n}\right) \in \mathbb{S}^{\infty} \times \mathbb{H}_{B M O}^{2} \times$ $\mathbb{J}_{B M O}^{2}$ for each $n \geq 1$. Furthermore, as a special case of the universal bounds, one sees $\left\|Y^{m, n}\right\|_{\mathbb{S} \infty},\left\|Z^{m, n}\right\|_{\mathbb{H}_{B M O}^{2}},\left\|\psi^{m, n}\right\|_{\mathbb{J}_{B M O}^{2}} \leq C$ with $C=C\left(\|\xi\|_{\infty},\|l\|_{\mathbb{S} \infty}+\delta m, \beta, \gamma, T\right)$.

Let denote $\delta Y^{m, n}:=Y^{m, n}-Y^{m, n-1}$. Replacing $l_{r}$ by $l_{r}+\delta m$, then putting $\delta=0$, and considering the drivers $f^{1}(r, y, z, \psi):=f_{m}\left(r,\left(Y_{v}^{m, n}\right)_{v \in[r, T]}, y, z, \psi\right), \quad f^{2}(r, y, z, \psi):=$ $f_{m}\left(r,\left(Y_{v}^{m, n-1}\right)_{v \in[r, T]}, y, z, \psi\right)$, one sees that $\left(f^{i}\right)_{i=1}^{2}$ satisfy Assumptions 3.1 and 3.2. Thus one can apply the stability results in Proposition 3.1 to the BSDE (6.2). In particular, by (3.8), one has for any $p \geq 2 q_{*}$ and $0<h \leq T$,

$$
\begin{aligned}
\mathbb{E}\left[\sup _{t \in[T-h, T]}\left|\delta Y_{t}^{m, n+1}\right|^{p}\right] \leq C \mathbb{E}\left[\left(\int_{T-h}^{T} \mathbb{E}_{\mathcal{F}_{r}} \mid f_{m}\left(r,\left(Y_{v}^{m, n}\right)_{v \in[r, T]}, \Theta_{r}^{m, n+1}\right)\right.\right. \\
\left.\left.\quad-f_{m}\left(r,\left(Y_{v}^{m, n-1}\right)_{v \in[r, T]}, \Theta_{r}^{m, n+1}\right) \mid d r\right)^{p}\right] \leq C h^{p} \mathbb{E}\left[\sup _{t \in[T-h, T]}\left|\delta Y_{t}^{m, n}\right|^{p}\right]
\end{aligned}
$$


with some constant $C=C\left(p, K .,\|\xi\|_{\infty},\|l\|_{\mathbb{S} \infty}+\delta m, \beta, \gamma, T\right)$. By choosing $h$ small enough so that $C h^{p}<1$, it becomes a strict contraction and thus $\left(Y_{v}^{m, n}, v \in[T-h, T]\right)_{n \geq 1}$ forms a Cauchy sequence in $\mathbb{S}^{p}[T-h, T]$.

By extracting an appropriate subsequence $\left(n^{\prime}\right) \subset(n)$, one has $\left\|\delta Y^{m, n^{\prime}}\right\|_{\mathbb{S}^{\infty}[T-h, T]} \rightarrow 0$ as $n^{\prime} \rightarrow \infty$. Applying Ito formula to $\left(\delta Y^{m, n^{\prime}}\right)^{2}$ and repeating the same procedures used in last part of the proof in Theorem 4.1, one can show that $\exists\left(Y^{m}, Z^{m}, \psi^{m}\right) \in\left(\mathbb{S}^{\infty} \times \mathbb{H}_{B M O}^{2} \times\right.$ $\left.\mathbb{J}_{B M O}^{2}\right)_{[T-h, T]},\left(Y^{m, n^{\prime}}, Z^{m, n^{\prime}}, \psi^{m, n^{\prime}}\right) \rightarrow\left(Y^{m}, Z^{m}, S^{m}\right)$ in the corresponding norm, and that $\left(Y_{v}^{m}, Z_{v}^{m}, \psi_{v}^{m}\right)_{v \in[T-h, T]}$ solves the ABSDE (6.1) for the period $[T-h, T]$ 卢

Now, let us replace $\left(Y^{m, n}, Z^{m, n}, \psi^{m, n}\right)_{n \in \mathbb{N}}$ by $\left(Y^{m}, Z^{m}, \psi^{m}\right)$ for $(\omega, s) \in \Omega \times[T-h, T]$ in (6.2). Then for $t \leq T-h$, we have

$$
\begin{aligned}
& Y_{t}^{m, n}=Y_{T-h}^{m}+\int_{t}^{T-h} \mathbb{E}_{\mathcal{F}_{r}} f_{m}\left(r,\left(Y_{v}^{m, n-1}\right)_{v \in[r, T]}, Y_{r}^{m, n}, Z_{r}^{m, n}, \psi_{r}^{m, n}\right) d r \\
& -\int_{t}^{T-h} Z_{r}^{m, n} d W_{r}-\int_{t}^{T-h} \int_{E} \psi_{r}^{m, n}(e) \widetilde{\mu}(d r, d e) .
\end{aligned}
$$

An application of Proposition 3.1 with the data $\left(Y_{T-h}^{m}, f^{1}\right),\left(Y_{T-h}^{m}, f^{2}\right)$ yields,

$$
\begin{aligned}
\mathbb{E}\left[\sup _{t \in[T-2 h, T-h]}\left|\delta Y^{m, n+1}\right|^{p}\right] & \leq C \mathbb{E}\left[\left(\int_{T-2 h}^{T-h} \mathbb{E}_{\mathcal{F}_{r}}|\delta f(r)| d r\right)^{p}\right] \\
& \leq C h^{p} \mathbb{E}\left[\sup _{t \in[T-2 h, T]}\left|\delta Y_{t}^{m, n}\right|^{p}\right]=C h^{p} \mathbb{E}\left[\sup _{t \in[T-2 h, T-h]}\left|\delta Y_{t}^{m, n}\right|^{p}\right]
\end{aligned}
$$

where the fact $Y_{s}^{m, n}=Y_{s}^{m}, s \in[T-h, T]$ is used in the 2nd line. Thus one can extend the solution to the period $[T-2 h, T-h]$ by the same procedures used in the previous step. Since coefficient $C$ can be taken independently of the specific period, the whole period $[0, T]$ can be covered by a finite number of partitions. Notice here that, as one can see from the proof of Proposition 3.1, the coefficient $C$ depends on the essential supremum of the terminal value $\|\xi\|_{\infty}$ only through the local Lipschitz constant $K_{M}$ and the universal bounds controlling $M$ as well as the coefficients of the reverse Hölder inequality. Hence the appearance of the new terminal value $Y_{T-h}^{m}$ does not change the size of the coefficient $C$. This finishes the proof for the existence of a bounded solution to (6.1) for each $m$.

\subsection{Comparison principle}

For completeness, we give a sufficient condition for the comparison principle to hold for our ABSDE in the rest of this section. In non-anticipated settings, i.e. when there is no future path-dependence of $\left(Y_{v}\right)_{v \in[0, T]}$ in the driver $f$, it is known that the comparison principle holds

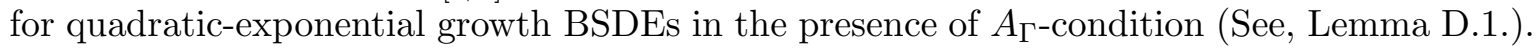
For the current anticipated setting, we need an additional assumption same as the one used in Theorem 5.1 of [32]. Consider the two ABSDEs with $i \in\{1,2\}$,

$$
Y_{t}^{i}=\xi_{i}+\int_{t}^{T} \mathbb{E}_{\mathcal{F}_{r}} f_{i}\left(r,\left(Y_{v}^{i}\right)_{v \in[r, T]}, Y_{r}^{i}, Z_{r}^{i}, \psi_{r}^{i}\right) d r-\int_{t}^{T} Z_{r}^{i} d W_{r}-\int_{t}^{T} \int_{E} \psi_{r}^{i}(e) \widetilde{\mu}(d r, d e)
$$

for $t \in[0, T]$.

Theorem 6.2. Suppose the data $\left(\xi_{i}, f_{i}\right)_{1 \leq i \leq 2}$ satisfy Assumptions 3.1, 3.2 and 6.1. Moreover, $f_{2}$ is increasing in $\left(q_{v}\right)_{v \in[0, T]}$, i.e. $f_{2}\left(r,\left(q_{v}\right)_{v \in[r, T]}, y, z, \psi\right) \leq f_{2}\left(r,\left(q_{v}^{\prime}\right)_{v \in[r, T]}, y, z, \psi\right)$ for every

\footnotetext{
${ }^{5}$ Thanks to the uniqueness of the solution of (6.1), the same arguments used in Remark 4.3 guarantee that the above convergence actually occurs in the entire sequence $(n)$.
} 
$(r, y, z, \psi) \in[0, T] \times \mathbb{R} \times \mathbb{R}^{1 \times d} \times \mathbb{L}^{2}(E, \nu)$ and $q, q^{\prime} \in \mathbb{D}[0, T]$, if $q_{v} \leq q_{v}^{\prime} \forall v \in[r, T]$. If $\xi_{1} \leq \xi_{2}$ a.s. and $f_{1}\left(r,\left(q_{v}\right)_{v \in[r, T]}, y, z, \psi\right) \leq f_{2}\left(r,\left(q_{v}\right)_{v \in[r, T]}, y, z, \psi\right) d \mathbb{P} \otimes d r$-a.e. for every $(q, y, z, \psi) \in \mathbb{D}[0, T] \times \mathbb{R} \times \mathbb{R}^{1 \times d} \times \mathbb{L}^{2}(E, \nu)$, then $Y_{t}^{1} \leq Y_{t}^{2} \forall t \in[0, T]$ a.s.

Proof. Firstly, let us regularize the driver $f_{2}$ by $f_{2}^{\prime}$ defined as, for every $(r, q, y, z, \psi)$,

$$
f_{2}^{\prime}\left(r,\left(q_{v}\right)_{r \in[t, T]}, y, z, \psi\right):=f_{2}\left(r,\left(\varphi_{m}\left(q_{v}\right)\right)_{v \in[r, T]}, y, z, \psi\right)
$$

with some truncation level $m$ satisfying $m>\left(\left\|Y^{1}\right\|_{\mathbb{S} \infty} \vee\left\|Y^{2}\right\|_{\mathbb{S} \infty}\right)$. Consider a sequence of non-anticipated BSDEs with $n \in \mathbb{N}$ by

$$
\begin{aligned}
Y_{t}^{2, n}= & \xi_{2}+\int_{t}^{T} \mathbb{E}_{\mathcal{F}_{r}} f_{2}^{\prime}\left(r,\left(Y_{v}^{2, n-1}\right)_{v \in[r, T]}, Y_{r}^{2, n}, Z_{r}^{2, n}, \psi_{r}^{2, n}\right) d r \\
& -\int_{t}^{T} Z_{r}^{2, n} d W_{r}-\int_{t}^{T} \int_{E} \psi_{r}^{2, n}(e) \widetilde{\mu}(d r, d e), \quad t \in[0, T]
\end{aligned}
$$

under the condition $Y^{2,0}=Y^{1}$. By the proof of Theorem 6.1, there exists $h>0$ such that $\left(Y^{2, n}, Z^{2, n}, \psi^{2, n}\right) \rightarrow\left(Y^{2}, Z^{2}, \psi^{2}\right)$ in $\mathbb{S}^{\infty} \times \mathbb{H}_{B M O}^{2} \times \mathbb{J}_{B M O}^{2}$ as $n \rightarrow \infty$ for the period $[T-h, T]$. Note that the constraint $\varphi_{m}(\cdot)$ becomes passive at least for large enough $n$.

Firstly, let us focus on the period $[T-h, T]$. Set $\widetilde{f}_{1}(r, y, z, \psi)=\mathbb{E}_{\mathcal{F}_{r}} f_{1}\left(r,\left(Y_{v}^{1}\right)_{v \in[r, T]}, y, z, \psi\right)$ and $\widetilde{f}_{2}(r, y, z, \psi)=\mathbb{E}_{\mathcal{F}_{r}} f_{2}^{\prime}\left(r,\left(Y_{v}^{1}\right)_{v \in[r, T]}, y, z, \psi\right)=\mathbb{E}_{\mathcal{F}_{r}} f_{2}\left(r,\left(Y_{v}^{1}\right)_{v \in[r, T]}, y, z, \psi\right)$. Applying Lemma D.1, one obtains $Y_{t}^{1}=Y_{t}^{2,0} \leq Y_{t}^{2,1} \forall t \in[T-h, T]$ a.s. Then using the new definition

$$
\begin{aligned}
& \widetilde{f}_{1}(r, y, z, \psi)=\mathbb{E}_{\mathcal{F}_{r}} f_{2}^{\prime}\left(r,\left(Y_{v}^{2,0}\right)_{v \in[r, T]}, y, z, \psi\right), \\
& \widetilde{f}_{2}(r, y, z, \psi)=\mathbb{E}_{\mathcal{F}_{r}} f_{2}^{\prime}\left(r,\left(Y_{v}^{2,1}\right)_{v \in[r, T]}, y, z, \psi\right),
\end{aligned}
$$

and the hypothesis that the driver is increasing in $q \in \mathbb{D}[0, T]$, Lemma D.1 yields $Y_{t}^{2,1} \leq$ $Y^{2,2} \forall t \in[T-h, T]$ a.s. By repeating the same arguments, one sees $Y_{t}^{1} \leq Y_{t}^{2, n-1} \leq Y_{t}^{2, n} \forall t \in$ $[T-h, T]$ a.s. for every $n \in \mathbb{N}$. Since $Y^{2, n}$ converges to $Y^{2}$ in $\mathbb{S}^{\infty}[T-h, T]$, one concludes $Y_{t}^{1} \leq Y_{t}^{2} \forall t \in[T-h, T]$ a.s.

Let us now replace $Y_{t}^{2, n}$ by $Y_{t}^{2}$ for all $t \in[T-h, T]$ in (6.4), and consider a sequence of non-anticipated BSDEs $n \in \mathbb{N}$

$$
\begin{aligned}
Y_{t}^{2, n}= & Y_{T-h}^{2}+\int_{t}^{T-h} \mathbb{E}_{\mathcal{F}_{r}} f_{2}^{\prime}\left(r,\left(Y_{v}^{2, n-1}\right)_{v \in[r, T]}, Y_{r}^{2, n}, Z_{r}^{2, n}, \psi_{r}^{2, n}\right) d r \\
& -\int_{t}^{T-h} Z_{r}^{2, n} d W_{r}-\int_{t}^{T-h} \int_{E} \psi_{r}^{2, n}(e) \widetilde{\mu}(d r, d e)
\end{aligned}
$$

with the initial condition $Y_{t}^{2,0}=\left\{\begin{array}{l}Y_{t}^{1}, t \in[0, T-h) \\ Y_{t}^{2}, t \in[T-h, T]\end{array}\right.$ for the next short period $t \in[T-$ $2 h, T-h]$. By the result of the previous step, one has $Y_{t}^{1} \leq Y_{t}^{2,0} \forall t \in[T-2 h, T]$ a.s. Now, let us set $\widetilde{f}_{1}(r, y, z, \psi)=\mathbb{E}_{\mathcal{F}_{r}} f_{1}\left(r,\left(Y_{v}^{1}\right)_{v \in[r, T]}, y, z, \psi\right), \widetilde{f}_{2}(r, y, z, \psi)=\mathbb{E}_{\mathcal{F}_{r}} f_{2}^{\prime}\left(r,\left(Y_{v}^{2,0}\right)_{v \in[r, T]}, y, z, \psi\right)$, where the latter is equal to $\mathbb{E}_{\mathcal{F}_{r}} f_{2}\left(r,\left(Y_{v}^{2,0}\right)_{v \in[r, T]}, y, z, \psi\right)$. By applying Lemma D.1 to the data $\left(Y_{T-h}^{1}, \tilde{f}_{1}\right),\left(Y_{T-h}^{2}, \widetilde{f}_{2}\right)$, one obtains $Y_{t}^{1} \leq Y_{t}^{2,1} \forall t \in[T-2 h, T-h]$ a.s. Since $Y_{t}^{2,1}=Y_{t}^{2}$ for $t \in[T-h, T]$, one concludes $Y_{t}^{1} \leq Y_{t}^{2,0} \leq Y_{t}^{2,1} \forall t \in[T-2 h, T]$ a.s. Similarly, applying Lemma D.1 with $\widetilde{f}_{1}(r, y, z, \psi)=\mathbb{E}_{\mathcal{F}_{r}} f_{2}^{\prime}\left(r,\left(Y_{v}^{2, n-2}\right)_{v \in[r, T]}, y, z, \psi\right), \widetilde{f}_{2}(r, y, z, \psi)=$ $\mathbb{E}_{\mathcal{F}_{r}} f_{2}^{\prime}\left(r,\left(Y_{v}^{2, n-1}\right)_{v \in[r, T]}, y, z, \psi\right)$ yields $Y_{t}^{2, n-1} \leq Y_{t}^{2, n} \forall t \in[T-2 h, T]$ a.s. for every $n \geq 2$. As in the previous step, the proof of Theorem 6.1 implies $Y^{2, n} \rightarrow Y^{2}$ in $\mathbb{S}^{\infty}[T-2 h, T-h]$. Since 
$Y_{t}^{2, n}=Y_{t}^{2}$ for $t \in[T-h, T]$ by construction, one actually has $Y^{2, n} \rightarrow Y^{2}$ in $\mathbb{S}^{\infty}[T-2 h, T]$. It follows that $Y_{t}^{1} \leq Y_{t}^{2} \forall t \in[T-2 h, T]$ a.s. Repeating the same procedures finite number of times, one obtains the desired result.

\section{A Some preliminary results}

Let us remind some important properties of BMO-martingales. For our purpose, it is enough to focus on continuous ones. When $Z \in \mathbb{H}_{B M O}^{2}, M$. := $\int_{0}^{\cdot} Z_{r} d W_{r}$ is a continuous BMOmartingale with $\|M\|_{B M O}=\|Z\|_{\mathbb{H}_{B M O}^{2}}$.

Lemma A.1 (reverse Hölder inequality). Let $M$ be a continuous BMO-martingale. Then, Doléans-Dade exponential $\left(\mathcal{E}_{t}(M), t \in[0, T]\right)$ is a uniformly integrable martingale, and for every stopping time $\tau \in \mathcal{T}_{0}^{T}$, there exists some $r>1$ such that $\mathbb{E}\left[\mathcal{E}_{T}(M)^{r} \mid \mathcal{F}_{\tau}\right] \leq C \mathcal{E}_{\tau}(M)^{r}$ with some positive constant $C=C\left(r,\|M\|_{B M O}\right)$.

Proof. See Kazamaki (1979) [19, and also Remark 3.1 of Kazamaki (1994) 20].

Lemma A.2. Let $M$ be a square integrable continuous martingale and $\hat{M}:=\langle M\rangle-M$. Then, $M \in B M O(\mathbb{P})$ if and only if $\hat{M} \in B M O(\mathbb{Q})$ with $d \mathbb{Q} / d \mathbb{P}=\mathcal{E}_{T}(M)$. Furthermore, $\|\hat{M}\|_{B M O(\mathbb{Q})}$ is determined by some function of $\|M\|_{B M O(\mathbb{P})}$ and vice versa.

Proof. See Theorem 3.3 and Theorem 2.4 in [20].

Remark A.1. For continuous martingales, Theorem 3.1 [20] also tells that there exists some decreasing function $\Phi(r)$ with $\Phi(1+)=\infty$ and $\Phi(\infty)=0$ such that if $\|M\|_{B M O(\mathbb{P})}$ satisfies $\|M\|_{B M O(\mathbb{P})}<\Phi(r)$ then $\mathcal{E}(M)$ satisfies the reverse Hölder inequality with power $r$. This implies together with Lemma A.2, one can take a common positive constant $\bar{r}$ satisfying $1<\bar{r} \leq r^{*}$ such that both of the $\mathcal{E}(M)$ and $\mathcal{E}(\hat{M})$ satisfy the reverse Hölder inequality with power $\bar{r}$ under the respective probability measure $\mathbb{P}$ and $\mathbb{Q}$. Furthermore, the upper bound $r^{*}$ is determined only by $\|M\|_{B M O(\mathbb{P})}$ (or equivalently by $\|M\|_{B M O(\mathbb{Q})}$ ).

Let us also remind the following result.

Lemma A.3. (Chapter 1, Section 9, Lemma 6 [24]) For any $\Psi \in \mathbb{J}^{p}$ with $p \geq 2$, there exists some constant $C=C(p)$ such that

$$
\mathbb{E}\left[\left(\int_{0}^{T} \int_{E}\left|\Psi_{r}(e)\right|^{2} \nu(d e) d r\right)^{\frac{p}{2}}\right] \leq C \mathbb{E}\left[\left(\int_{0}^{T} \int_{E}\left|\Psi_{r}(e)\right|^{2} \mu(d r, d e)\right)^{\frac{p}{2}}\right] .
$$

Lemma A.4. (Lemma 5-1 of Bichteler, Gravereaux and Jacod (1987) [5]) Let $\eta: \mathbb{R} \rightarrow \mathbb{R}$ be defined by $\eta(e)=1 \wedge|e|$. Then, for $\forall p \geq 2$, there exists a constant $\delta_{p}$ depending on $p, T, n, k$ such that

$$
\mathbb{E}\left[\sup _{t \in[0, T]}\left|\int_{0}^{t} \int_{E} U(s, e) \widetilde{\mu}(d s, d e)\right|^{p}\right] \leq \delta_{p} \int_{0}^{T} \mathbb{E}\left|L_{s}\right|^{p} d s
$$

if $U$ is an $\mathbb{R}^{n \times k}$-valued $\mathcal{P} \otimes \mathcal{E}$-measurable function on $\Omega \times[0, T] \times E$ and $L$ is a predictable process satisfying $\left|U^{i}(\omega, s, e)\right| \leq L_{s}(\omega) \eta(e)$ for each column $1 \leq i \leq k$.

\section{B Technical details omitted in the main text}

In the main text, we have omitted some technical details in order not to interrupt the main story. In this section, let us give the omitted details for completeness. 


\section{B.1 Details of the proof of Lemma 3.1}

By assumption, we have $Y \in \mathbb{S}^{\infty}$. Since $\|\psi\|_{J^{\infty}} \leq 2\|Y\|_{\mathbb{S}^{\infty}}, \psi$ is bounded. Ito formula applied to $e^{2 \gamma Y_{t}}$ yields, for any $\mathbb{F}$-stopping time $\tau \in \mathcal{T}_{0}^{T}$,

$$
\begin{aligned}
& \mathbb{E}_{\mathcal{F}_{\tau}}\left[\int_{\tau}^{T} e^{2 \gamma Y_{s}} 2 \gamma^{2}\left|Z_{s}\right|^{2} d s+\int_{\tau}^{T} \int_{E} e^{2 \gamma Y_{s}}\left(e^{\gamma \psi_{s}(e)}-1\right)^{2} \nu(d e) d s\right] \\
& =\mathbb{E}_{\mathcal{F}_{\tau}}\left[e^{2 \gamma Y_{T}}-e^{2 \gamma Y_{\tau}}+2 \gamma \int_{\tau}^{T} e^{2 \gamma Y_{s}}\left(\mathbb{E}_{\mathcal{F}_{s}} f\left(s,\left(Y_{v}\right)_{v \in[s, T]}, Y_{s}, Z_{s}, \psi_{s}\right)-\int_{E} j_{\gamma}\left(\psi_{s}(e)\right) \nu(d e)\right) d s\right] \\
& \leq \mathbb{E}_{\mathcal{F}_{\tau}}\left[e^{2 \gamma Y_{T}}-e^{2 \gamma Y_{\tau}}+2 \gamma \int_{\tau}^{T} e^{2 \gamma Y_{s}}\left(l_{s}+\delta\|Y\|_{[s, T]}+\beta\left|Y_{s}\right|+\frac{\gamma}{2}\left|Z_{s}\right|^{2}\right) d s\right]
\end{aligned}
$$

where structure condition in Assumption 3.1 was used in the third line. Then it yields

$$
\begin{aligned}
& \mathbb{E}_{\mathcal{F}_{\tau}}\left[\int_{\tau}^{T} e^{2 \gamma Y_{s}} \gamma^{2}\left|Z_{s}\right|^{2} d s+\int_{\tau}^{T} \int_{E} e^{2 \gamma Y_{s}}\left(e^{\gamma \psi_{s}(e)}-1\right)^{2} \nu(d e) d s\right] \\
& \leq e^{2 \gamma\|Y\|_{\mathbb{S} \infty}}+2 \gamma e^{2 \gamma\|Y\|_{\mathbb{S} \infty}} T\left(\|l\|_{\mathbb{S} \infty}+(\beta+\delta)\|Y\|_{\mathbb{S} \infty}\right) .
\end{aligned}
$$

Since $e^{-2 \gamma\|Y\|_{\mathbb{S} \infty}} \leq e^{ \pm 2 \gamma Y} \leq e^{2 \gamma\|Y\|_{\mathbb{S}^{\infty}}}$,

$$
\begin{aligned}
& \mathbb{E}_{\mathcal{F}_{\tau}}\left[\int_{\tau}^{T} \gamma^{2}\left|Z_{s}\right|^{2} d s+\int_{\tau}^{T} \int_{E}\left(e^{\gamma \psi_{s}(e)}-1\right)^{2} \nu(d e) d s\right] \\
& \leq e^{4 \gamma\|Y\|_{\mathbb{S} \infty}}+2 \gamma e^{4 \gamma\|Y\|_{\mathbb{S} \infty}} T\left(\|l\|_{\mathbb{S} \infty}+(\beta+\delta)\|Y\|_{\mathbb{S} \infty}\right) .
\end{aligned}
$$

In particular, this leads to the desired bound on $\|Z\|_{\mathbb{H}_{B M O}^{2}}^{2}$.

Repeating the same calculation on $e^{-2 \gamma Y_{t}}$, one obtains the next estimate:

$$
\begin{aligned}
& \mathbb{E}_{\mathcal{F}_{\tau}}\left[\int_{\tau}^{T} \gamma^{2}\left|Z_{s}\right|^{2} d s+\int_{\tau}^{T} \int_{E}\left(e^{-\gamma \psi_{s}(e)}-1\right)^{2} \nu(d e) d s\right] \\
& \leq e^{4 \gamma\|Y\|_{\mathbb{S} \infty}}+2 \gamma e^{4 \gamma\|Y\|_{\mathbb{S} \infty}} T\left(\|l\|_{\mathbb{S} \infty}+(\beta+\delta)\|Y\|_{\mathbb{S} \infty}\right) .
\end{aligned}
$$

Noticing the fact that $\left(e^{x}-1\right)^{2}+\left(e^{-x}-1\right)^{2} \geq x^{2}, \forall x \in \mathbb{R}$, one obtains

$$
\|\psi\|_{\mathbb{J}_{B}^{2}}^{2} \leq \frac{e^{4 \gamma\|Y\|_{\mathbb{S} \infty}}}{\gamma^{2}}\left(2+4 \gamma T\left(\|l\|_{\mathbb{S} \infty}+(\beta+\gamma)\|Y\|_{\mathbb{S} \infty}\right)\right) .
$$

Finally, the relation

$$
\|\psi\|_{\mathbb{J}_{B M O}^{2}}^{2} \leq\|\psi\|_{\mathbb{J}_{B}^{2}}^{2}+\|\psi\|_{\mathbb{J}^{\infty}}^{2} \leq\|\psi\|_{\mathbb{J}_{B}^{2}}^{2}+4\|Y\|_{\mathbb{S}^{\infty}}^{2}
$$

proves the desired estimate on $\|\psi\|_{\mathbb{J}_{B M O}^{2}}^{2}$. 


\section{B.2 Derivation of (3.4)}

Using (3.3), one gets

$$
\begin{aligned}
d P_{t}= & P_{t-} \gamma d\left(e^{\beta t}\left|Y_{t}\right|+\int_{0}^{t} e^{\beta r}\left(l_{r}+\delta \mathbb{E}_{\mathcal{F}_{r}} \sup _{v \in[r, T]}\left|Y_{v}\right|\right) d r\right)+P_{t} \frac{\gamma^{2}}{2}\left|e^{\beta t} \operatorname{sign}\left(Y_{t}\right) Z_{t}\right|^{2} d t \\
& +P_{t-} \int_{E}\left(e^{\gamma e^{\beta t}\left(\left|Y_{t-}+\psi_{t}(e)\right|-\left|Y_{t-}\right|\right)}-1-\gamma e^{\beta t} \operatorname{sign}\left(Y_{t-}\right) \psi_{t}(e)\right) \mu(d t, d e) \\
=P_{t-} & \int_{E}\left(e^{\gamma e^{\beta t}\left(\left|Y_{t-}+\psi_{t}(e)\right|-\left|Y_{t-}\right|\right)}-1-\gamma e^{\beta t} \operatorname{sign}\left(Y_{t-}\right) \psi_{t}(e)\right) \mu(d t, d e) \\
& +P_{t} \frac{\gamma^{2}}{2}\left|e^{\beta t} \operatorname{sign}\left(Y_{t}\right) Z_{t}\right|^{2} d t+P_{t-\gamma}\left\{e^{\beta t} \operatorname{sign}\left(Y_{t}\right) Z_{t} d W_{t}+\int_{E} e^{\beta t} \operatorname{sign}\left(Y_{t-}\right) \psi_{t}(e) \widetilde{\mu}(d t, d e)\right. \\
& \left.-\int_{E} j_{\gamma}\left(e^{\beta t} \operatorname{sign}\left(Y_{t}\right) \psi_{t}(e)\right) \nu(d e) d t-\frac{\gamma}{2}\left|e^{\beta t} \operatorname{sign}\left(Y_{t}\right) Z_{t}\right|^{2} d t+d C_{t}\right\} .
\end{aligned}
$$

Separating the terms contained in $d C^{\prime}(3.5)$ and canceling $|Z|^{2}$-term, one obtains

$$
\begin{aligned}
& d P_{t}=P_{t-}\left\{\gamma d C_{t}+\int_{E}\left(e^{\gamma e^{\beta t}\left(\left|Y_{t-}+\psi_{t}(e)\right|-\left|Y_{t-}\right|\right)}-e^{\gamma e^{\beta t} \operatorname{sign}\left(Y_{t-}\right) \psi_{t}(e)}\right) \mu(d t, d e)\right\} \\
& +P_{t-} \int_{E}\left(e^{\gamma e^{\beta t} \operatorname{sign}\left(Y_{t-}\right) \psi_{t}(e)}-1-\gamma e^{\beta t} \operatorname{sign}\left(Y_{t-}\right) \psi_{t}(e)\right) \mu(d t, d e) \\
& +P_{t-} \gamma\left\{e^{\beta t} \operatorname{sign}\left(Y_{t}\right) Z_{t} d W_{t}+\int_{E} e^{\beta t} \operatorname{sign}\left(Y_{t-}\right) \psi_{t}(e) \widetilde{\mu}(d t, d e)-\int_{E} j_{\gamma}\left(e^{\beta t} \operatorname{sign}\left(Y_{t}\right) \psi_{t}(e)\right) \nu(d e) d t\right\} .
\end{aligned}
$$

Notice that the terms inside a parenthesis in the second line are equal to $\gamma j_{\gamma}\left(e^{\beta t} \operatorname{sign}\left(Y_{t-}\right) \psi_{t}(e)\right)$, which then yields

$$
\begin{aligned}
d P_{t} & =P_{t-} d C_{t}^{\prime}+P_{t-} \int_{E} \gamma j_{\gamma}\left(e^{\beta t} \operatorname{sign}\left(Y_{t-}\right) \psi_{t}(e)\right) \widetilde{\mu}(d t, d e) \\
& +P_{t-}\left\{\gamma e^{\beta t} \operatorname{sign}\left(Y_{t}\right) Z_{t} d W_{t}+\int_{E} \gamma e^{\beta t} \operatorname{sign}\left(Y_{t-}\right) \psi_{t}(e) \widetilde{\mu}(d t, d e)\right\} .
\end{aligned}
$$

Using the definition of $j_{\gamma}(\cdot)$, one obtains the desired expression (3.4).

\section{B.3 The proof for Lemma 4.1}

The existence of unique solution $X^{t, x} \in \mathbb{S}^{p}, \forall p \geq 2$ and $\forall(t, x) \in[0, T] \times \mathbb{R}^{n}$ is well known for the Lipschitz SDEs with jumps. Hence, we only provide a proof for the relevant continuities below.

(a) For any $s \in[t, T]$ and $p \geq 2$, the BDG inequality yields

$$
\begin{aligned}
\mathbb{E}\left[\left|X_{s}^{t, x}\right|^{p}\right] & \leq C \mathbb{E}\left\{|x|^{p}+\left(\int_{t}^{s}\left|b\left(r, X_{r}^{t, x}\right)\right| d r\right)^{p}+\left(\int_{t}^{s}\left|\sigma\left(r, X_{r}^{t, x}\right)\right|^{2} d r\right)^{\frac{p}{2}}\right. \\
& \left.+\sup _{u \in[t, s]}\left|\int_{t}^{u} \int_{E}\right| \gamma\left(r, X_{r-}^{t, x}, e\right)|\widetilde{\mu}(d r, d e)|^{p}\right\}
\end{aligned}
$$

Since for each $1 \leq i \leq k$, we have $\left|\gamma^{i}\left(r, X_{r-}^{t, x}, e\right)\right| \leq K\left(1+\left|X_{r-}^{t, x}\right|\right) \eta(e)$ by Assumption 4.1 (ii) and (iii). By Lemma A.4 and the Lipschitz continuity yields,

$$
\mathbb{E}\left[\left|X_{s}^{t, x}\right|\right] \leq C\left(1+|x|^{p}\right)+C \int_{t}^{s} \mathbb{E}\left[\left|X_{r}^{t, x}\right|^{p}\right] d r
$$


and hence the Gronwall inequality gives $\sup _{s \in[t, T]} \mathbb{E}\left[\left|X_{s}^{t, x}\right|^{p}\right] \leq C\left(1+|x|^{p}\right)$. Noticing the fact that $X_{s}^{t, x} \equiv x$ for $s \leq t$ and applying BDG inequality once again, one obtains

$$
\mathbb{E}\left[\sup _{s \in[0, T]}\left|X_{s}^{t, x}\right|^{p}\right] \leq C\left(1+|x|^{p}\right) .
$$

(b) Let us assume $t \leq s \leq u \leq s+h$. The case with $s<t$ can be done similarly by using $X_{s}^{t, x} \equiv x$ for $s \leq t$. Since

$$
X_{u}^{t, x}-X_{s}^{t, x}=\int_{s}^{u} b\left(r, X_{r}^{t, x}\right) d r+\int_{s}^{u} \sigma\left(r, X_{r}^{t, x}\right) d W_{r}+\int_{s}^{u} \int_{E} \gamma\left(r, X_{r-}^{t, x}\right) \widetilde{\mu}(d r, d e) .
$$

Using the BDG inequality, Lemma A.4 and the result (a), one obtains

$$
\begin{aligned}
\mathbb{E}\left[\sup _{u \in[s, s+h]}\left|X_{u}^{t, x}-X_{s}^{t, x}\right|^{p}\right] & \leq C\left(1+\mathbb{E}\left[\sup _{r \in[t, T]}\left|X_{r}^{t, x}\right|^{p} \mid\right]\right) h \\
& \leq C\left(1+|x|^{p}\right) h
\end{aligned}
$$

which gives the desired result.

(c) Without loss of generality, we assume $0 \leq t^{\prime} \leq t \leq T$. We separate the problem into the three cases with respect to the range of $s$. Firstly, we clearly have

$$
\mathbb{E} \sup _{0 \leq s \leq t^{\prime}}\left|X_{s}^{t, x}-X_{s}^{t^{\prime}, x^{\prime}}\right|^{p} \leq\left|x-x^{\prime}\right|^{p} .
$$

Secondly, let us consider

$$
\begin{aligned}
& \mathbb{E} \sup _{t^{\prime} \leq s \leq t}\left|X_{s}^{t, x}-X_{s}^{t^{\prime}, x^{\prime}}\right|^{p}=\mathbb{E} \sup _{t^{\prime} \leq s \leq t}\left|x-X_{s}^{t^{\prime}, x^{\prime}}\right|^{p} \\
& \leq C \mathbb{E} \sup _{t^{\prime} \leq s \leq t}\left(\left|x^{\prime}-X_{s}^{t^{\prime}, x^{\prime}}\right|^{p}+\left|x-x^{\prime}\right|^{p}\right) \\
& \leq C\left(\left|x-x^{\prime}\right|^{p}+\left(1+\left|x^{\prime}\right|^{p}\right)\left|t-t^{\prime}\right|\right)
\end{aligned}
$$

where, in the last inequality, we have used the result (b).

Finally, we consider the case $s \geq t$. Note that

$$
X_{s}^{t^{\prime}, x^{\prime}}=X_{t}^{t^{\prime}, x^{\prime}}+\int_{t}^{s} b\left(r, X_{r}^{t^{\prime}, x^{\prime}}\right) d r+\int_{t}^{s} \sigma\left(r, X_{r}^{t^{\prime}, x^{\prime}}\right) d W_{r}+\int_{t}^{s} \int_{E} \gamma\left(r, X_{r-}^{t^{\prime}, x^{\prime}}, e\right) \widetilde{\mu}(d r, d e)
$$

and hence

$$
\begin{aligned}
X_{s}^{t, x}-X_{s}^{t^{\prime}, x^{\prime}} & =x-x^{\prime}-\left(X_{t}^{t^{\prime}, x^{\prime}}-x^{\prime}\right) \\
& +\int_{t}^{s}\left[b\left(r, X_{r}^{t, x}\right)-b\left(r, X_{r}^{t^{\prime}, x^{\prime}}\right)\right] d r+\int_{t}^{s}\left[\sigma\left(r, X_{r}^{t, x}\right)-\sigma\left(r, X_{r}^{t^{\prime}, x^{\prime}}\right)\right] d W_{r} \\
& +\int_{t}^{s} \int_{E}\left[\gamma\left(r, X_{r-}^{t, x}, e\right)-\gamma\left(r, X_{r-}^{t^{\prime}, x^{\prime}}, e\right)\right] \widetilde{\mu}(d r, d e) .
\end{aligned}
$$


Applying BDG inequality and Lemma A.4, one obtains

$$
\begin{aligned}
& \mathbb{E}\left[\sup _{s \in[t, T]}\left|X_{s}^{t, x}-X_{s}^{t^{\prime}, x^{\prime}}\right|^{p}\right] \leq C \mathbb{E}\left\{\left|x-x^{\prime}\right|^{p}+\left|X_{t}^{t^{\prime}, x^{\prime}}-x^{\prime}\right|^{p}\right. \\
& +\left(\int_{t}^{T}\left|b\left(r, X_{r}^{t, x}\right)-b\left(r, X_{r}^{t^{\prime}, x^{\prime}}\right)\right| d r\right)^{p}+\left(\int_{t}^{T}\left|\sigma\left(r, X_{r}^{t, x}\right)-\sigma\left(r, X_{r}^{t^{\prime}, x^{\prime}}\right)\right|^{2} d r\right)^{p / 2} \\
& \left.+\int_{t}^{T}\left|X_{r}^{t, x}-X_{r}^{t^{\prime}, x^{\prime}}\right| p d r\right\} \\
& \leq C\left(\left|x-x^{\prime}\right|^{p}+\left(1+\left|x^{\prime}\right|^{p}\right)\left|t-t^{\prime}\right|\right)+C \int_{t}^{T} \mathbb{E}\left[\sup _{s \in[r, T]}\left|X_{s}^{t, x}-X_{s}^{t^{\prime}, x^{\prime}}\right|^{p}\right] d r
\end{aligned}
$$

where, in the last inequality, the result (b) was used.

Using the backward Gronwall inequality, one obtains

$$
\mathbb{E}\left[\sup _{s \in[t, T]}\left|X_{s}^{t, x}-X_{s}^{t^{\prime}, x^{\prime}}\right|^{p}\right] \leq C\left(\left|x-x^{\prime}\right|^{p}+\left(1+\left|x^{\prime}\right|^{p}\right)\left|t-t^{\prime}\right|\right) .
$$

Adding the above three cases and flipping the role of $t, t^{\prime}$, one obtains in general

$$
\mathbb{E}\left[\sup _{s \in[0, T]}\left|X_{s}^{t, x}-X_{s}^{t^{\prime}, x^{\prime}}\right|^{p}\right] \leq C\left(\left|x-x^{\prime}\right|^{p}+\left(1+\left(|x| \vee\left|x^{\prime}\right|\right)^{p}\right)\left|t-t^{\prime}\right|\right) .
$$

\section{Existence and uniqueness results for Lipschitz case}

Anticipated BSDEs under the global Lipschitz condition have been studied by many authors. Our setup is a bit different from the standard one, in particular at the terminal condition and also at the point where the continuity of the driver is defined with respect to the uniform norm of the path rather than $\mathbb{L}^{2}[0, T]$-norm. For readers' convenience, we provide a proof under our particular setup. It is restricted to the simplest form relevant for our purpose. One can readily generalize it to multi-dimensional setups with the future $(Z, \psi)$-dependence (See [28] among others.).

Let us consider the ABSDE for $t \in[0, T]$

$$
Y_{t}=\xi+\int_{t}^{T} \mathbb{E}_{\mathcal{F}_{r}} f\left(r,\left(Y_{v}\right)_{v \in[r, T]}, Y_{r}, Z_{r}, \psi_{r}\right) d r-\int_{t}^{T} Z_{r} d W_{r}-\int_{t}^{T} \int_{E} \psi_{r}(e) \widetilde{\mu}(d r, d e)
$$

where $f: \Omega \times[0, T] \times \mathbb{D}[0, T] \times \mathbb{R} \times \mathbb{R}^{1 \times d} \times \mathbb{L}^{2}(E, \nu) \rightarrow \mathbb{R}$ and $\xi$ is an $\mathcal{F}_{T}$-measurable random variable.

Assumption C.1. (i) The driver $f$ is a map such that for every $(y, z, \psi) \in \mathbb{R} \times \mathbb{R}^{1 \times d} \times$ $\mathbb{L}^{2}(E, \nu)$ and any càdlàg $\mathbb{F}$-adapted process $\left(Y_{v}\right)_{v \in[0, T]}$, the process $\left(\mathbb{E}_{\mathcal{F}_{t}} f\left(t,\left(Y_{v}\right)_{v \in[t, T]}, y, z, \psi\right), t \in\right.$ $[0, T])$ is progressively measurable.

(ii) For every $(q, y, z, \psi),\left(q^{\prime}, y^{\prime}, z^{\prime}, \psi^{\prime}\right) \in \mathbb{D}[0, T] \times \mathbb{R} \times \mathbb{R}^{1 \times d} \times \mathbb{L}^{2}(E, \nu)$, there exists some positive constant $K$ such that

$$
\begin{aligned}
& \left|f\left(t,\left(q_{v}\right)_{v \in[t, T]}, y, z, \psi\right)-f\left(t,\left(q_{v}^{\prime}\right)_{v \in[t, T]}, y^{\prime}, z^{\prime}, \psi^{\prime}\right)\right| \\
& \quad \leq K\left(\sup _{v \in[t, T]}\left|q_{v}-q_{v}^{\prime}\right|+\left|y-y^{\prime}\right|+\left|z-z^{\prime}\right|+|| \psi-\psi^{\prime}||_{\mathbb{L}^{2}(\nu)}\right)
\end{aligned}
$$

$d \mathbb{P} \otimes d t$-a.e. $(\omega, t) \in \Omega \times[0, T]$.

(iii) $\mathbb{E}\left[|\xi|^{2}+\left(\int_{0}^{T}|f(r, 0,0,0,0)| d r\right)^{2}\right]<\infty$. 
Proposition C.1. Under Assumption C.1, there exists a unique solution $(Y, Z, \psi) \in \mathbb{S}^{2} \times$ $\mathbb{H}^{2} \times \mathbb{J}^{2}$ to the ABSDE (C.1).

Proof. We prove the claim by constructing a strictly contracting map $\Phi: \mathcal{K}^{2}[0, T] \ni\left(Y^{k}, Z^{k}, \psi^{k}\right) \mapsto$ $\Phi\left(Y^{k}, Z^{k}, \psi^{k}\right)=:\left(Y^{k+1}, Z^{k+1}, \psi^{k+1}\right) \in \mathcal{K}^{2}[0, T]$ defined by

$Y_{t}^{k+1}=\xi+\int_{t}^{T} \mathbb{E}_{\mathcal{F}_{r}} f\left(r,\left(Y_{v}^{k}\right)_{v \in[r, T]}, Y_{r}^{k}, Z_{r}^{k}, \psi_{r}^{k}\right) d r-\int_{t}^{T} Z_{r}^{k+1} d W_{r}-\int_{t}^{T} \int_{E} \psi_{r}^{k+1}(e) \widetilde{\mu}(d r, d e)$

with $k \in \mathbb{N}_{0}$ and $\left(Y^{0}, Z^{0}, \psi^{0}\right) \equiv(0,0,0)$. It is easy to see that the map is well-defined. Let $\delta Y^{k+1}:=Y^{k+1}-Y^{k}, \quad \delta Z^{k+1}:=Z^{k+1}-Z^{k}, \quad \delta \psi^{k+1}:=\psi^{k+1}-\psi^{k}, \quad \Theta^{k}:=\left(Y^{k}, Z^{k}, \psi^{k}\right)$.

We consider the norm $\|\cdot\|_{\mathcal{K}_{\beta}^{2}}$ equivalent to $\|\cdot\|_{\mathcal{K}^{2}}$ defined with some $\beta>0$

$$
\|(Y, Z, \psi)\|_{\mathcal{K}_{\beta}^{2}}^{2}:=\mathbb{E}\left[\sup _{r \in[0, T]}\left|e^{\beta r} Y_{r}\right|^{2}\right]+\mathbb{E} \int_{0}^{T}\left|e^{\beta r} Z_{r}\right|^{2} d r+\mathbb{E} \int_{0}^{T}\left\|e^{\beta r} \psi_{r}\right\|_{\mathbb{L}^{2}(\nu)}^{2} d r
$$

Applying Ito formula to $e^{2 \beta t}\left|\delta Y_{t}^{k+1}\right|^{2}$, one obtains for any $t \in[0, T]$

$$
\begin{aligned}
& e^{2 \beta t}\left|\delta Y_{t}^{k+1}\right|^{2}+\int_{t}^{T} e^{2 \beta r}\left|\delta Z_{r}^{k+1}\right|^{2} d r+\int_{t}^{T} \int_{E} e^{2 \beta r}\left|\delta \psi_{r}^{k+1}(e)\right|^{2} \mu(d r, d e) \\
& =\int_{t}^{T} e^{2 \beta r}\left(2 \delta Y_{r}^{k+1} \mathbb{E}_{\mathcal{F}_{r}}\left[f\left(r,\left(Y_{v}^{k}\right)_{v \in[r, T]}, \Theta_{r}^{k}\right)-f\left(r,\left(Y_{v}^{k-1}\right)_{v \in[r, T]}, \Theta_{r}^{k-1}\right)\right]-2 \beta\left|\delta Y_{r}^{k+1}\right|^{2}\right) d r \\
& -\int_{t}^{T} e^{2 \beta r} 2 \delta Y_{r}^{k+1} \delta Z_{r}^{k+1} d W_{r}-\int_{t}^{T} \int_{E} e^{2 \beta r} 2 \delta Y_{r-}^{k+1} \delta \psi_{r}^{k+1}(e) \widetilde{\mu}(d r, d e)
\end{aligned}
$$

For any $\epsilon>0$, one has

$$
\begin{aligned}
& 2 \delta Y_{r}^{k+1} \mathbb{E}_{\mathcal{F}_{r}}\left[f\left(r,\left(Y_{v}^{k}\right)_{v \in[r, T]}, \Theta_{r}^{k}\right)-f\left(r,\left(Y_{v}^{k-1}\right)_{v \in[r, T]}, \Theta_{r}^{k-1}\right)\right]-2 \beta\left|\delta Y_{r}^{k+1}\right|^{2} \\
& \leq 2 K\left|\delta Y_{r}^{k+1}\right|\left(2 \mathbb{E}_{\mathcal{F}_{r}}\left[\left\|\delta Y^{k}\right\|_{[r, T]}\right]+\left|\delta Z_{r}^{k}\right|+\left\|\delta \psi_{r}^{k}\right\|_{\mathbb{L}^{2}(\nu)}\right)-2 \beta\left|\delta Y_{r}^{k+1}\right|^{2} \\
& \leq\left(\frac{6 K^{2}}{\epsilon}-2 \beta\right)\left|\delta Y_{r}^{k+1}\right|^{2}+\epsilon\left(\mathbb{E}_{\mathcal{F}_{r}}\left[|| \delta Y^{k} \|_{[r, T]}^{2}\right]+\left|\delta Z_{r}^{k}\right|^{2}+\left\|\delta \psi_{r}^{k}\right\|_{\mathbb{L}^{2}(\nu)}^{2}\right) .
\end{aligned}
$$

Thus, choosing $\beta=\beta(\epsilon)=3 K^{2} / \epsilon$ and taking expectation with $t=0$ yields

$$
\left\|e^{\beta \cdot} \delta Z^{k+1}\right\|_{\mathbb{H}^{2}}^{2}+\left\|e^{\beta \cdot} \delta \psi^{k+1}\right\|_{\mathbb{J}^{2}}^{2} \leq \epsilon\left(T\left\|e^{\beta \cdot} \delta Y^{k}\right\|_{\mathbb{S}^{2}}^{2}+\left\|e^{\beta \cdot \delta} \delta Z^{k}\right\|_{\mathbb{H}^{2}}^{2}+\left\|e^{\beta \cdot} \delta \psi^{k}\right\|_{\mathbb{J}^{2}}^{2}\right)
$$

Next, let us apply the BDG inequality (Theorem 48 in IV.4. of [33]) to (C.2). Then there exists some constant $C$ such that

$$
\begin{aligned}
& \mathbb{E}\left[\left\|e^{\beta \cdot} \delta Y^{k+1}\right\|_{[0, T]}^{2}\right] \leq \epsilon\left(T\left\|e^{\beta \cdot} \delta Y^{k}\right\|_{\mathbb{S}^{2}}^{2}+\left\|e^{\beta \cdot \delta} \delta Z^{k}\right\|_{\mathbb{H}^{2}}^{2}+\left\|e^{\beta \cdot} \delta \psi^{k}\right\|_{\mathbb{J}^{2}}^{2}\right) \\
& +C \mathbb{E}\left[\left(\int_{0}^{T}\left|e^{\beta r} \delta Y_{r}^{k+1}\right|^{2}\left|e^{\beta r} \delta Z_{r}^{k+1}\right|^{2} d r\right)^{\frac{1}{2}}\right]+C \mathbb{E}\left[\left(\int_{0}^{T} \int_{E}\left|e^{\beta r} \delta Y_{r-}^{k+1}\right|^{2}\left|e^{\beta r} \delta \psi_{r}^{k+1}(e)\right|^{2} \mu(d r, d e)\right)^{\frac{1}{2}}\right] \\
& \leq \epsilon\left(T\left\|e^{\beta \cdot} \delta Y^{k}\right\|_{\mathbb{S}^{2}}^{2}+\left\|e^{\beta \cdot \delta} \delta Z^{k}\right\|_{\mathbb{H}^{2}}^{2}+\left\|e^{\beta \cdot} \delta \psi^{k}\right\|_{\mathbb{J}^{2}}^{2}\right)+\frac{1}{2} \mathbb{E}\left[\left\|e^{\beta \cdot} \delta Y^{k+1}\right\|_{[0, T]}^{2}\right] \\
& +C\left(\left\|e^{\beta \cdot} \delta Z^{k+1}\right\|_{\mathbb{H}^{2}}^{2}+\left\|e^{\beta \cdot} \delta \psi^{k+1}\right\|_{\mathbb{J}^{2}}^{2}\right) .
\end{aligned}
$$


Thus, with some constant $C$ (which is independent of $\epsilon, \beta$ ),

$$
\left\|e^{\beta \cdot} \delta Y^{k+1}\right\|_{\mathbb{S}^{2}}^{2} \leq 2 \epsilon\left(T\left\|e^{\beta \cdot} \delta Y^{k}\right\|_{\mathbb{S}^{2}}^{2}+\left\|e^{\beta \cdot \delta} \delta Z^{k}\right\|_{\mathbb{H}^{2}}^{2}+\left\|e^{\beta \cdot} \delta \psi^{k}\right\|_{\mathbb{J}^{2}}^{2}\right)+C\left(\left\|e^{\beta \cdot} \delta Z^{k+1}\right\|_{\mathbb{H}^{2}}^{2}+\left\|e^{\beta \cdot} \delta \psi^{k+1}\right\|_{\mathbb{J}^{2}}^{2}\right) .
$$

Combining with (C.3), one obtains

$$
\left\|\left(\delta Y^{k+1}, \delta Z^{k+1}, \delta \psi^{k+1}\right)\right\|_{\mathcal{K}_{\beta(\epsilon)}^{2}}^{2} \leq \epsilon(C+3)(T \vee 1)\left\|\left(\delta Y^{k}, \delta Z^{k}, \delta \psi^{k}\right)\right\|_{\mathcal{K}_{\beta(\epsilon)}^{2}}^{2}
$$

and hence by choosing $\epsilon$ so that $\epsilon(C+3)(T \vee 1)<1$ (and $\beta(\epsilon)$ accordingly) makes the map $\Phi$ strict contraction with respect to the norm $\mathcal{K}_{\beta(\epsilon)}^{2}$. This proves the existence as well as the uniqueness.

\section{Comparison principle for non-anticipated settings}

Consider the two BSDEs with $i=\{1,2\}$,

$$
Y_{t}^{i}=\xi_{i}+\int_{t}^{T} \widetilde{f}_{i}\left(r, Y_{r}^{i}, Z_{r}^{i}, \psi_{r}^{i}\right) d r-\int_{t}^{T} Z_{r}^{i} d W_{r}-\int_{t}^{T} \int_{E} \psi_{r}^{i}(e) \widetilde{\mu}(d r, d e)
$$

for $t \in[0, T]$.

Lemma D.1. Suppose $\left(\xi, \widetilde{f}_{i}\right)_{1 \leq i \leq 2}$ satisfy Assumptions 3.1, 3.2 and 4.1 of [16], which correspond to Assumptions [3.1, 3.2 and 6.1 of the current paper without the $Y$ 's future path dependence, respectively. If $\xi_{1} \leq \xi_{2}$ a.s. and $\tilde{f}_{1}(r, y, z, \psi) \leq \widetilde{f}_{2}(r, y, z, \psi) d \mathbb{P} \otimes d r$-a.e. for every $(y, z, \psi) \in \mathbb{R} \times \mathbb{R}^{1 \times d} \times \mathbb{L}^{2}(E, \nu)$, then $Y_{t}^{1} \leq Y_{t}^{2} \forall t \in[0, T]$ a.s.

Proof. One can prove it in the same way as Theorem 2.5 of [35]. By Theorem 4.1 [16], there exists a unique solution $\left(Y^{i}, Z^{i}, \psi^{i}\right)_{1 \leq i \leq 2} \in \mathbb{S}^{\infty} \times \mathbb{H}_{B M O}^{2} \times \mathbb{J}_{B M O}^{2}$ to the BSDEs (D.1) satisfying the universal bounds. Let us put $\delta Y:=Y^{1}-Y^{2}, \delta Z:=Z^{1}-Z^{2}, \delta \psi:=\psi^{1}-$ $\psi^{2}, \delta \widetilde{f}(r):=\left(\widetilde{f}_{1}-\widetilde{f}_{2}\right)\left(r, Y_{r}^{1}, Z_{r}^{1}, \psi_{r}^{1}\right)$. We also introduce the two progressively measurable processes $\left(a_{r}\right)_{r \in[0, T]},\left(b_{r}\right)_{r \in[0, T]}$ given by

$a_{r}:=\frac{\widetilde{f}_{2}\left(r, Y_{r}^{1}, Z_{r}^{1}, \psi_{r}^{1}\right)-\widetilde{f}_{2}\left(r, Y_{r}^{2}, Z_{r}^{1}, \psi_{r}^{1}\right)}{\delta Y_{r}} \mathbf{1}_{\delta Y_{r} \neq 0}, \quad b_{r}:=\frac{\tilde{f}_{2}\left(r, Y_{r}^{2}, Z_{r}^{1}, \psi_{r}^{1}\right)-\widetilde{f}_{2}\left(r, Y_{r}^{2}, Z_{r}^{2}, \psi_{r}^{1}\right)}{\left|\delta Z_{r}\right|^{2}} \mathbf{1}_{\delta Z_{r} \neq 0} \delta Z_{r}^{\top}$.

Note that $a \in \mathbb{S}^{\infty}$ and $b \in \mathbb{H}_{B M O}^{2}$ due to the universal bounds and the local Lipschitz

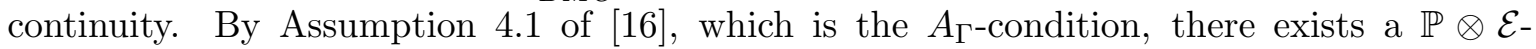
measurable process $\Gamma$ such that

$$
\begin{aligned}
\delta Y_{t} \leq & \delta \xi+\int_{t}^{T}\left(\delta \tilde{f}(r)+a_{r} \delta Y_{r}+b_{r} Z_{r}+\int_{\mathbb{E}} \Gamma_{r}(e) \delta \psi_{r}(e) \nu(d e)\right) d r \\
& -\int_{t}^{T} \delta Z_{r} d W_{r}-\int_{t}^{T} \int_{E} \delta \psi_{r}(e) \widetilde{\mu}(d r, d e)
\end{aligned}
$$

satisfying $C_{1}(1 \wedge|e|) \leq|\Gamma(e)| \leq C_{2}(1 \wedge|e|)$ with some constant $C_{1}>-1$ and $C_{2} \geq 0$. Here the fact that $Y^{i} \in \mathbb{S}^{\infty}, \psi^{i} \in \mathbb{J}^{\infty}$ was used. Since $\mathcal{M}:=\int_{0}^{\cdot} b_{r}^{\top} d W_{r}+\int_{0}^{\cdot} \int_{E} \Gamma_{r}(e) \widetilde{\mu}(d r, d e)$ is a BMOmartingale with jump size strictly bigger than -1 , one can define an equivalent measure $\mathbb{Q}$ by $d \mathbb{Q} / d \mathbb{P}=\mathcal{E}_{T}(\mathcal{M})$. Thus one obtains from (D.2)

$$
\delta Y_{t} \leq \mathbb{E}_{\mathcal{F}_{t}}^{\mathbb{Q}}\left[e^{R_{t, T}} \delta \xi+\int_{t}^{T} e^{R_{t, r}} \delta \widetilde{f}(r) d r\right]
$$


with $R_{t, s}:=\int_{t}^{s} a_{r} d r$. This proves the claim.

\section{Acknowledgement}

The research is partially supported by Center for Advanced Research in Finance (CARF).

\section{References}

[1] Antonelli, F., and Mancini, C., 2016, Solutions of BSDE's with jumps and quadratic/locally Lipschitz generator, Stochastic Processes and their Applications, 126, pp. 3124-3144.

[2] Barles, G., Buckdahn, R. and Pardoux, E., 1997, Backward stochastic differential equations and integral-partial differential equations, Stochastics and Stochastics Reports, Vol. 60 , pp. $57-83$.

[3] Barrieu, P. and El Karoui, N., 2013, Monotone stability of quadratic semimartingales with applications to unbounded general quadratic BSDEs, The Annals of Probability, Vol. 41, No. 3B, 1831-1863.

[4] Becherer, D., 2006, Bounded solutions to backward SDE's with jumps for utility optimization and indifference pricing, The Annals of Applied Probability, Vol. 16, No. 4, 2027-2054.

[5] Bichteler, K., Gravereaux, J. and Jacod, J., 1987, Malliavin calculus for processes with jumps, Stochastics Monographs, Gordon and Breach science publishers, LN.

[6] Bismut, J.M., 1973, Conjugate convex functions in optimal stochastic control, J. Math. Anal. Apl. 44, 384-404.

[7] Cohen, S. and Elliott, R., 2015 Quadratic BSDEs in Stochastic Calculus and Applications (2nd edition), Appendix A.9, 619-634. Birkhäuser, Springer NY.

[8] Cvitanić, J. and Zhang, J., 2013, Contract theory in continuous-time methods, Springer, Berlin.

[9] Delong, L., 2013, Backward Stochastic Differential Equations with Jumps and Their Actuarial and Financial Applications, Springer-Verlag, LN.

[10] El Karoui, N., Matoussi, A. and Ngoupeyou, A., 2016, Quadratic Exponential Semimartingales and Application to BSDE's with jumps, arXiv:1603.0691.

[11] El Karoui, N., Peng, S. and Quenez, M.C., 1997, Backward Stochastic Differential Equations in Finance, Mathematical Finance, Vol. 7, No. 1, 1-71.

[12] Epstein, L. and Zin, S., 1989, Substitution, risk aversion and temporal behavior of consumption and asset returns: A theoretical framework, Econometrica, 57, 937-969.

[13] Evans, L.C., 2010, Partial Differential Equations (second edition), Graduate Studies in Mathematics, Vol. 19, American Mathematical Society.

[14] Fromm, A. and Imkeller, P., 2013, Existence, uniqueness and regularity of decoupling fields to multidimensional fully coupled FBSDEs, arXiv:1310.0499. 
[15] Fromm, A., 2014, Theory and applications of decoupling fields for forward-backward stochastic differential equations, Ph.D thesis, Humboldt-Universität zu Berlin.

[16] Fujii, M. and Takahashi, A., 2017, Quadratic-exponential growth BSDEs with jumps and their Malliavin's differentiability, Stochastic Processes and their Applications, In Press. https://doi.org/10.1016/j.spa.2017.09.002.

[17] He, S., Wang, J. and Yang, J., 1992, Semimartingale Theory and Stochastic Calculus, Science Press and CRC Press, Beijing, China.

[18] Jeanblanc, M., Lim, T. and Agram, N., 2016, Some existence results for advanced backward stochastic differential equations with a jump time, HAL archives.

[19] Kazamaki, N., 1979, A sufficient condition for the uniform integrability of exponential martingales, Math. Rep. Toyama Univ. 2, 1-11. MR-0542374.

[20] Kazamaki, N., 1994, Continuous exponential martingales and BMO, Lecture Notes in Mathematics, vol. 1579, Springer-Verlag, Berlin.

[21] Kazi-Tani, N., Possamai, D. and Zhou, C., Quadratic BSDEs with jumps: a fixed-point approach, Electronic Journal of Probability, 20, No. 66, 1-28.

[22] Klenke, A., 2014, Probability Theory (2nd edition), Springer, LN.

[23] Kobylanski, M., 2000, Backward stochastic differential equations and partial differential equations with quadratic growth, The annals of probability, Vol. 28, No. 2, 558-602.

[24] Liptser, R.Sh. and Shiryayev, A.N., 1989, Theory of Martingales, Kluwer Academic Publishers, Netherlands.

[25] Morlais, M-A., 2010, A new existence result for quadratic BSDEs with jumps with application to the utility maximization problem, Stochastic processes and their applications, 120, 1966-1995.

[26] Morlais, M-A, 2009, Utility maximization in a jump market model, Stochastics, Vol. 81, No. 1, 1-27.

[27] Ngoupeyou, A.B., 2010, Optimisation des portefeuilles d'actifs soumis au risque de défaut, Ph.D. Thesis, Université d'Evry.

[28] Oksendal, B., Sulem, A. and Zhang, T., 2011, Optimal control of stochastic delay equations and time-advanced backward stochastic differential equations, Adv. Appl. Prob., $43,572-596$.

[29] Pamen, O.M., 2015, Optimal control for stochastic delay systems under model uncertainty: a stochastic differential game approach, J Optim Theory Appl, 167, 998-1031.

[30] Pardoux, E. and Peng, S., 1990, Adapted solution of a backward stochastic differential equations, Systems Control Lett., 14, 55-61.

[31] Pardoux, E. and Rascanu, A., 2014, Stochastic differential equations, backward SDEs, partial differential equations, Springer International Publishing, Switzerland.

[32] Peng, S. and Yang, Z., 2009, Anticipated backward stochastic differential equations, The Annals of Probability, Vol. 37, No. 3, 877-902. 
[33] Protter, P., 2005, Stochastic Integration and Differential Equations: 2nd edition, version 2.1, Springer, NY.

[34] Royden, H.L. and Fitzpatrick, 2010, Real Analysis (fourth edition), Prentice Hall, U.S.

[35] Royer, M., 2006, Backward stochastic differential equations with jumps and related nonlinear expectations, Stochastic process and their applications, 116, 1358-1376.

[36] Yang, Z. and Elliott, R.J., 2013, Some properties of generalized anticipated backward stochastic differential equations, Electron. Commun. Probab. 18, No. 63, 1-10.

[37] Xu, X.M., 2011, Necessary and sufficient condition for the comparison theorem of multidimensional anticipated backward stochastic differential equations, Science China Mathematics, 5, No.2, 301-311. 